\title{
A Systematic Review on the Effects of Botanicals on Skeletal Muscle Health in Order to Prevent Sarcopenia
}

\author{
M. Rondanelli, ${ }^{1}$ A. Miccono, ${ }^{2}$ G. Peroni, ${ }^{1}$ F. Guerriero, ${ }^{3}$ P. Morazzoni, ${ }^{4}$ A. Riva, ${ }^{4}$ \\ D. Guido, ${ }^{1,5,6}$ and S. Perna ${ }^{1}$ \\ ${ }^{1}$ Department of Public Health, Experimental and Forensic Medicine, Section of Human Nutrition, Endocrinology and Nutrition Unit, \\ Azienda di Servizi alla Persona, University of Pavia, 27100 Pavia, Italy \\ ${ }^{2}$ Department of Clinical Sciences, Faculty of Medicine and Surgery, University of Milan, Milan, Italy \\ ${ }^{3}$ Azienda di Servizi alla Persona, Pavia, Italy \\ ${ }^{4}$ Research and Development Unit, Indena, 20139 Milan, Italy \\ ${ }^{5}$ Department of Brain and Behavioral Sciences, Medical and Genomic Statistics Unit, University of Pavia, 27100 Pavia, Italy \\ ${ }^{6}$ Department of Public Health, Experimental and Forensic Medicine, Biostatistics and Clinical Epidemiology Unit, \\ University of Pavia, 27100 Pavia, Italy
}

Correspondence should be addressed to S. Perna; simoneperna@hotmail.it

Received 13 November 2015; Revised 20 January 2016; Accepted 24 January 2016

Academic Editor: Hyunsu Bae

Copyright (C) 2016 M. Rondanelli et al. This is an open access article distributed under the Creative Commons Attribution License, which permits unrestricted use, distribution, and reproduction in any medium, provided the original work is properly cited.

\begin{abstract}
We performed a systematic review to evaluate the evidence-based medicine regarding the main botanical extracts and their nutraceutical compounds correlated to skeletal muscle health in order to identify novel strategies that effectively attenuate skeletal muscle loss and enhance muscle function and to improve the quality of life of older subjects. This review contains all eligible studies from 2010 to 2015 and included 57 publications. We focused our attention on effects of botanical extracts on growth and health of muscle and divided these effects into five categories: anti-inflammation, muscle damage prevention, antifatigue, muscle atrophy prevention, and muscle regeneration and differentiation.
\end{abstract}

\section{Introduction}

Sarcopenia is the loss of muscle protein mass and of muscle function and it occurs with increasing age, being a major component in the development of frailty [1]. It is a syndrome characterized by the progressive and generalized loss of skeletal muscle mass and strength with a risk of adverse outcomes such as physical disability, poor quality of life, and death [2]. Preventative diet, exercise, or treatment interventions particularly in middle-aged adults at the low end of the spectrum of muscle function may help to preserve mobility in later years and improve health span [3]. The therapeutic options for sarcopenia are unclear and constantly evolving. The most rational approach to delay the progression of sarcopenia is based on the combination of proper nutrition, possibly associated with the use of dietary supplements, and a regular exercise program [4]. Despite the major advantages offered by natural therapies with their long traditional use and poor physiological and psychological addiction as is commonly seen with conventional medicine [5], few studies have been performed on the topic of age-correlated pathologies of skeletal muscle. The aim of this review was to investigate the effectiveness of botanicals on skeletal muscle health focusing on possible therapeutics approaches to prevent sarcopenia.

\section{Materials and Methods}

The present systematic review was performed according to the steps by Egger et al. [6] (Table 1), as follows: (i) configuration of a working group: three operators skilled in clinical nutrition in the geriatric age, of whom one was acting as a methodological operator and two were participating as clinical operators; (ii) formulation of the revision question on the basis of considerations made in 
TABLE 1: Summary of methodology.

\begin{tabular}{|c|c|c|}
\hline Step & General activities & Specific activities \\
\hline Step 1 & $\begin{array}{l}\text { Configuration of a } \\
\text { working group }\end{array}$ & $\begin{array}{l}\text { Selection of three operators skilled in clinical nutrition: } \\
\text { (i) One as methodological operator } \\
\text { (ii) Two as clinical operators }\end{array}$ \\
\hline Step 2 & $\begin{array}{l}\text { Formulation of the } \\
\text { revision question }\end{array}$ & $\begin{array}{l}\text { Evaluation of the state of the art in metabolic and nutritional disorders of sarcopenia and their treatment } \\
\text { with botanicals }\end{array}$ \\
\hline Step 3 & $\begin{array}{l}\text { Identification of } \\
\text { relevant studies on } \\
\text { PubMed }\end{array}$ & $\begin{array}{l}\text { (a) Identification of the key words (sarcopenia, nutrients, and dietary supplement), allowing the definition } \\
\text { of the interest field of the documents to be searched, grouped in inverted commas (“...), and used } \\
\text { separately or in combination } \\
\text { (b) Use of the Boolean (a data type with only two possible values: true and false) AND operator, which } \\
\text { allows the establishment of logical relations among concepts } \\
\text { (c) Research modalities: advanced search } \\
\text { (d) Limits: papers published in the last } 20 \text { years; in vitro, animal, and humans studies; languages: English } \\
\text { (e) Manual search performed by the senior researchers experienced in clinical nutrition through the } \\
\text { revision of reviews and individual articles on sarcopenia in the elderly, published in journals qualified in } \\
\text { the Index Medicus }\end{array}$ \\
\hline Step 4 & $\begin{array}{l}\text { Analysis and } \\
\text { presentation of the } \\
\text { outcomes }\end{array}$ & $\begin{array}{l}\text { The data extrapolated from the revised studies was investigated in the form of a narrative review of the } \\
\text { reports and was collocated in tables }\end{array}$ \\
\hline
\end{tabular}

the abstract: "sarcopenia and muscle mass, use of botanic extracts during aging"; (iii) identification of relevant studies: a research strategy was planned, on PubMed and Scopus, as follows: (a) definition of the key words (sarcopenia, muscle mass, inflammation, antioxidants, botanical extracts, phytotherapy, muscle atrophy, muscle fatigue, Camellia sinensis, Vitis vinifera, Zingiber officinale, Citrus aurantium, and Panax quinquefolius), allowing the definition of the interest field of the documents to be searched, grouped in inverted commas (“..."), and used separately or in combination; (b) use of the Boolean AND operator, which allows the establishment of logical relationships among concepts; (c) research modalities: advanced search; (d) limits: papers published until June 2015; humans, animals, in vivo, and in vitro studies; languages: English; (e) manual search performed by the senior researchers, experienced in clinical nutrition, through the revision of reviews and research articles on sarcopenia in the elderly, published in qualified journals of the Index Medicus.

Analysis and presentation of the outcomes had been done as follows: the data extrapolated from the revised studies were summarized in Tables 2-6; in particular, for each study, we specified the author and year of publication, the plant and the active principles, the models used, the posology, and the main results obtained. The analyses were carried out in the form of a narrative review of the reports. The flow diagram of narrative review of the literature has been reported in Figure 1. As shown in Figure 1, we consider several effects of botanical extracts on growth and health of muscle; we divided these effects into five categories: anti-inflammatory activity, muscle damage prevention, antifatigue, muscle atrophy prevention, and muscle regeneration and differentiation. After this, we examined the typology of the studies, that is, in vitro, animals (mice, rats), or human, and we classified the human data according to the different condition such as postmenopausal women, athletes or others. Another key point was to identify the dosage of the extracts for each trial and the botanical compounds responsible for the activity. The use of the databases as PubMed or Scopus was determinant to enrich our review. At the end, we reported an analysis of all plants and their extracts that have a beneficial role in preventing sarcopenia or improve muscle health condition.

\section{Results}

3.1. Screening and Selection Process of Study. Of 120 articles identified, 57 studies met inclusion criteria (Figure 1), including 17 focused on anti-inflammation, 8 focused on muscle damage, 11 based on antifatigue effects, 7 based on muscle athorpy, and 15 based on muscle differentiation and regeneration (Figure 1).

As reported in Table 2, there are different effects on skeletal muscle for each botanical. At present, we evaluated in the literature over 70 different mechanisms of action.

3.2. Anti-Inflammatory Activity. This research has been carried out based on the keywords "skeletal muscle mass" and "inflammation" and "botanicals" or "plants" or "extracts"; 21 articles were sourced and 17 studies are taken into account. Among these papers, 3 studies are in in vitro setting, 4 in animals, 8 in humans, and two both in animals and in in vitro setting (Table 3).

Inflammation and oxidative stress induce muscle damage and muscle pain [18] and several botanicals (Phlebodium decamanum, Citrus aurantium, Coffea arabica, Zingiber officinale, Eugenia punicifolia, Panax ginseng, Go-sha-jinki-Gan, Vitis vinifera, and Curcuma longa L.) have a significant role in the prevention of this phenomenon. 


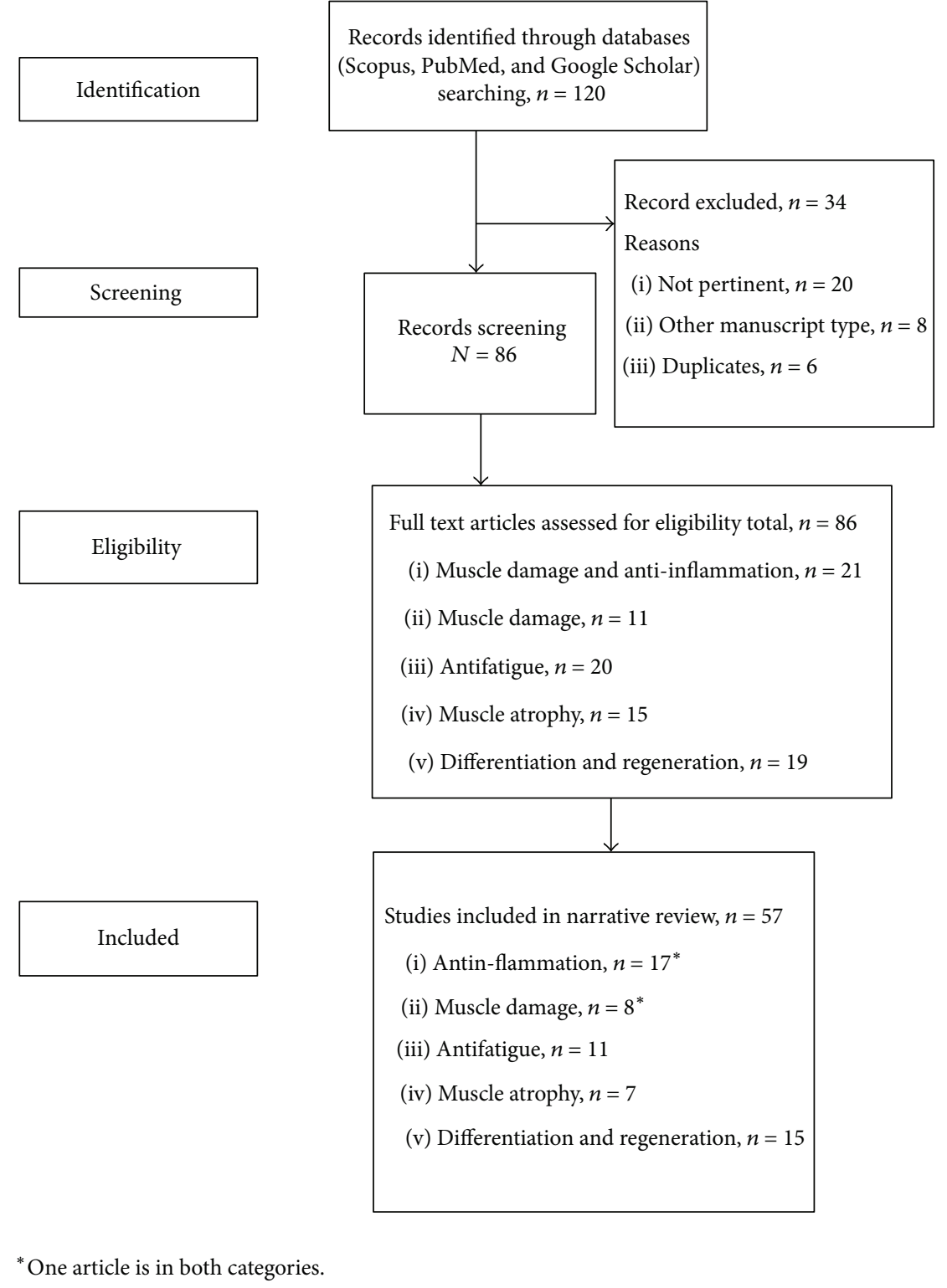

FIGURE 1: Flow diagram of narrative review of the literature.

Supplementation with Phlebodium decamanum (5 capsules of $400 \mathrm{mg}$ ) reduces inflammatory response and also the degree of oxidative stress in human during highintensity exercise, through the decrease of 8 -hydroxy-2 $2^{\prime}$ deoxyguanosine and isoprostanes generation, the increase of antioxidant enzyme activities in erythrocyte and total antioxidant status in plasma, the decrease of tumor necrosis factor (TNF- $\alpha$ ), and the increase of soluble receptor II of TNF- $\alpha$ (sTNF-RII), but kept the levels of interleukin-6 (IL6) and interleukin-1 antagonist receptor (IL-1ra) [18]. Other studies examine the anti-inflammatory effect of flavonoids isolated from Citrus aurantium, Coffea arabica, and Zingiber officinale on interleukins such as IL- $1 \alpha$ and IL- 6 and TNF$\alpha$ on skeletal muscle cells. Specifically, the flavonoids (hesperidin, nobiletin, and naringin of Citrus aurantium, also known as sour orange) inhibit the inflammatory response in lipopolysaccharide- (LPS-) induced L6 skeletal muscle cells. In addition, the flavonoids isolated from Korean Citrus aurantium $\mathrm{L}$. inhibit significantly inducible nitric oxide synthase (iNOS), cyclooxygenase-2 (COX-2), IL-6, and TNF$\alpha$ by blocking the nuclear factor-kappa B (NF- $\kappa \mathrm{b})$ and by blocking mitogen-activated protein kinases (MAPKs) signal pathways. Another study in the same muscle cells demonstrates the anti-inflammatory role of flavonoids isolated from Citrus aurantium through the modulation in protein related to the immune response. Furthermore, the pretreatment with flavonoids resulted in a decreased level of cleaved caspase3 , which is induced by muscle inflammation and is involved in muscle proteolysis and atrophy. Also, Zingiber officinale, commonly known as ginger, showed interesting antiinflammatory and analgesic effects in humans who ingested 2 grams of ginger or placebo after exercise; however, this extract 


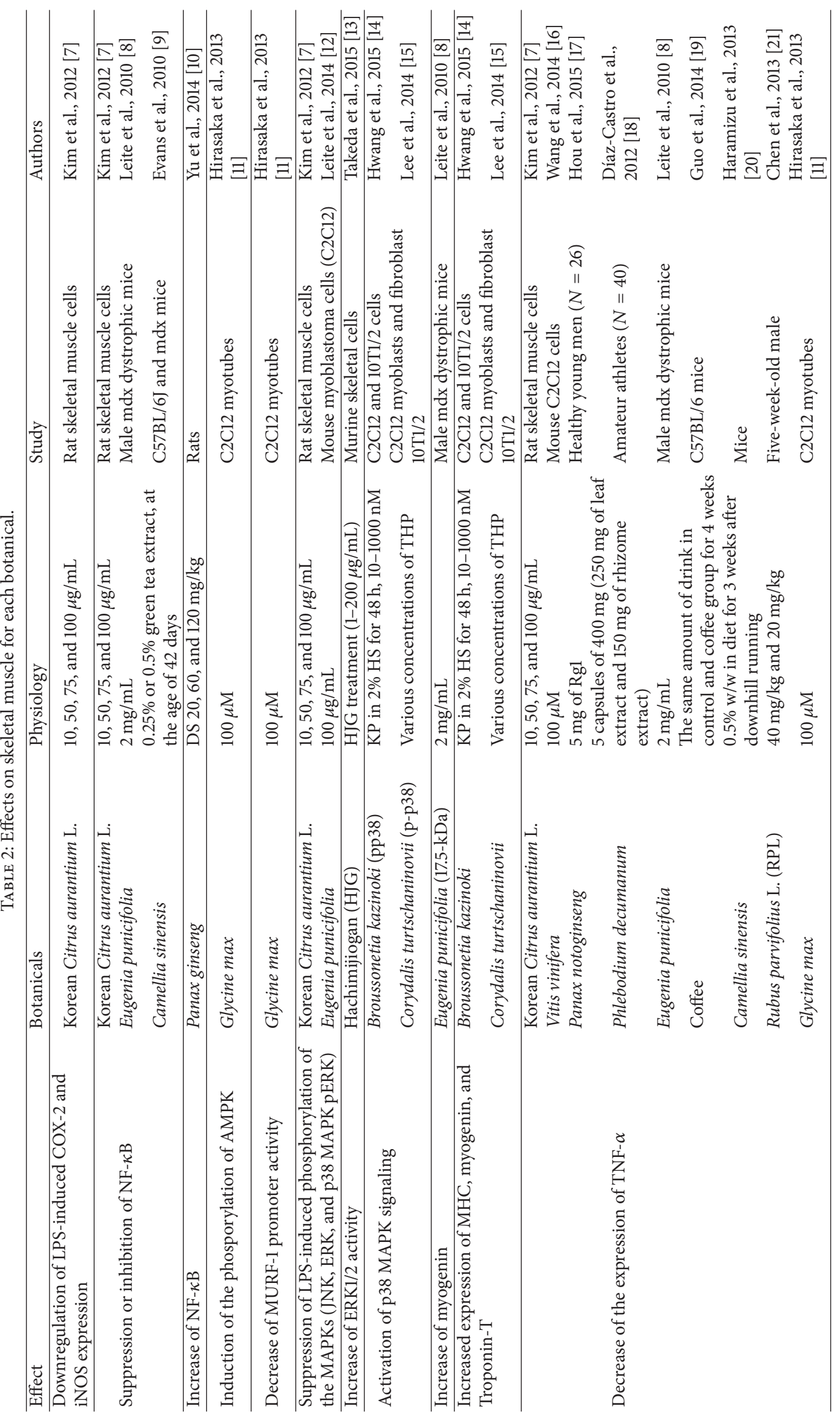




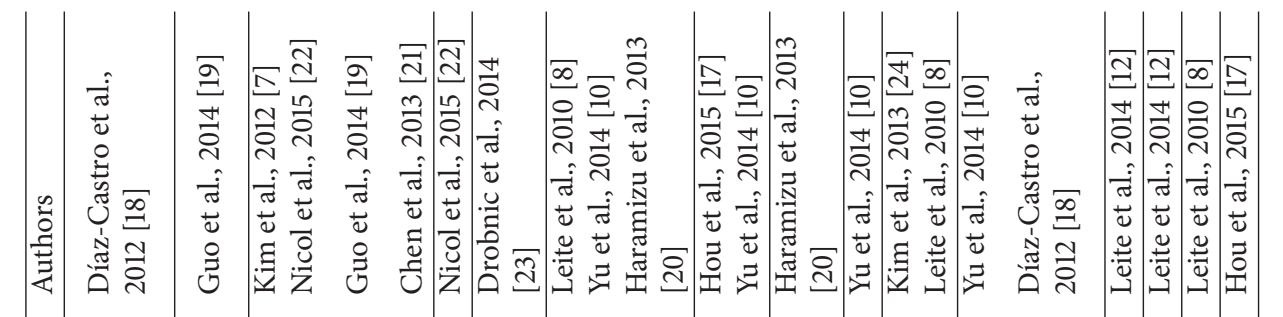




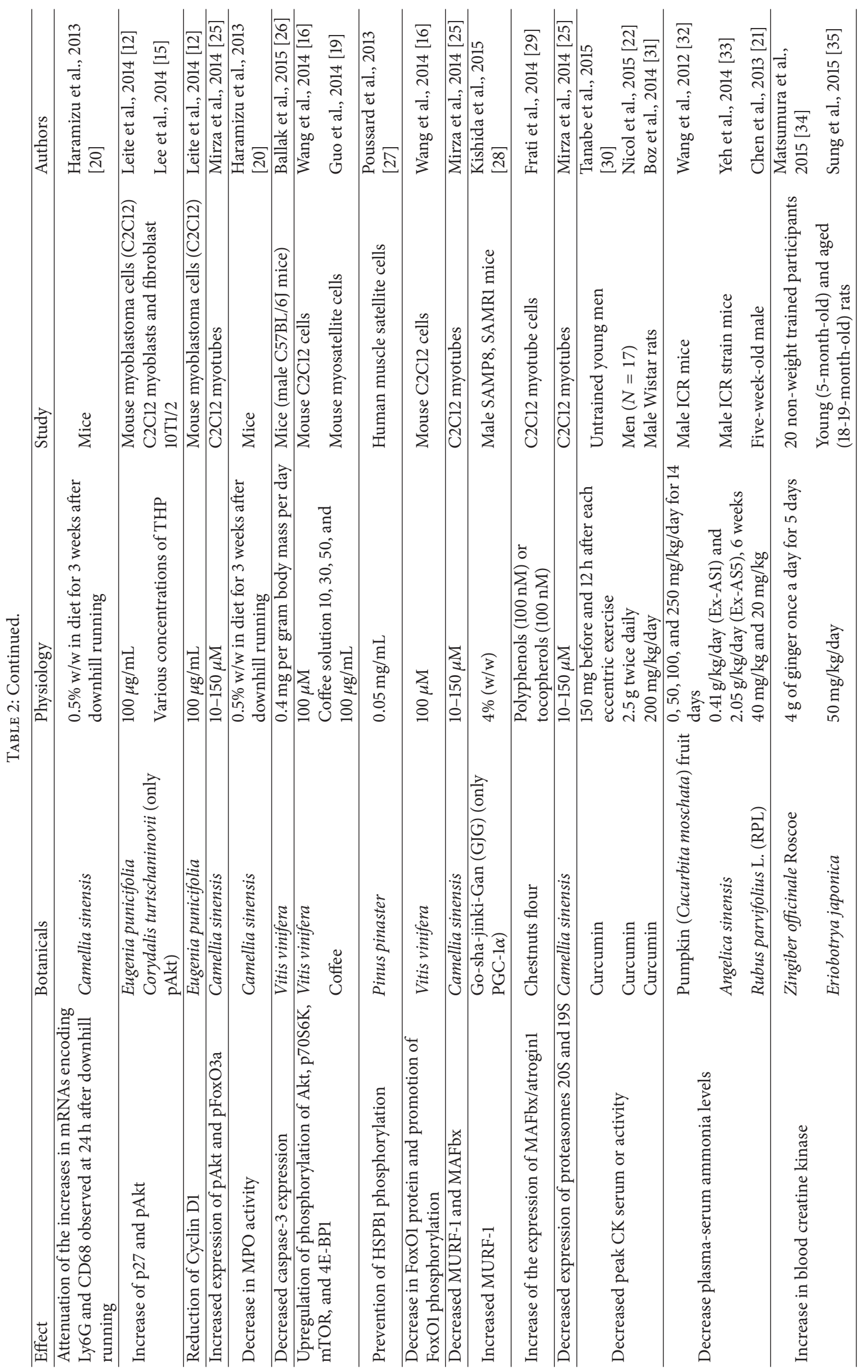




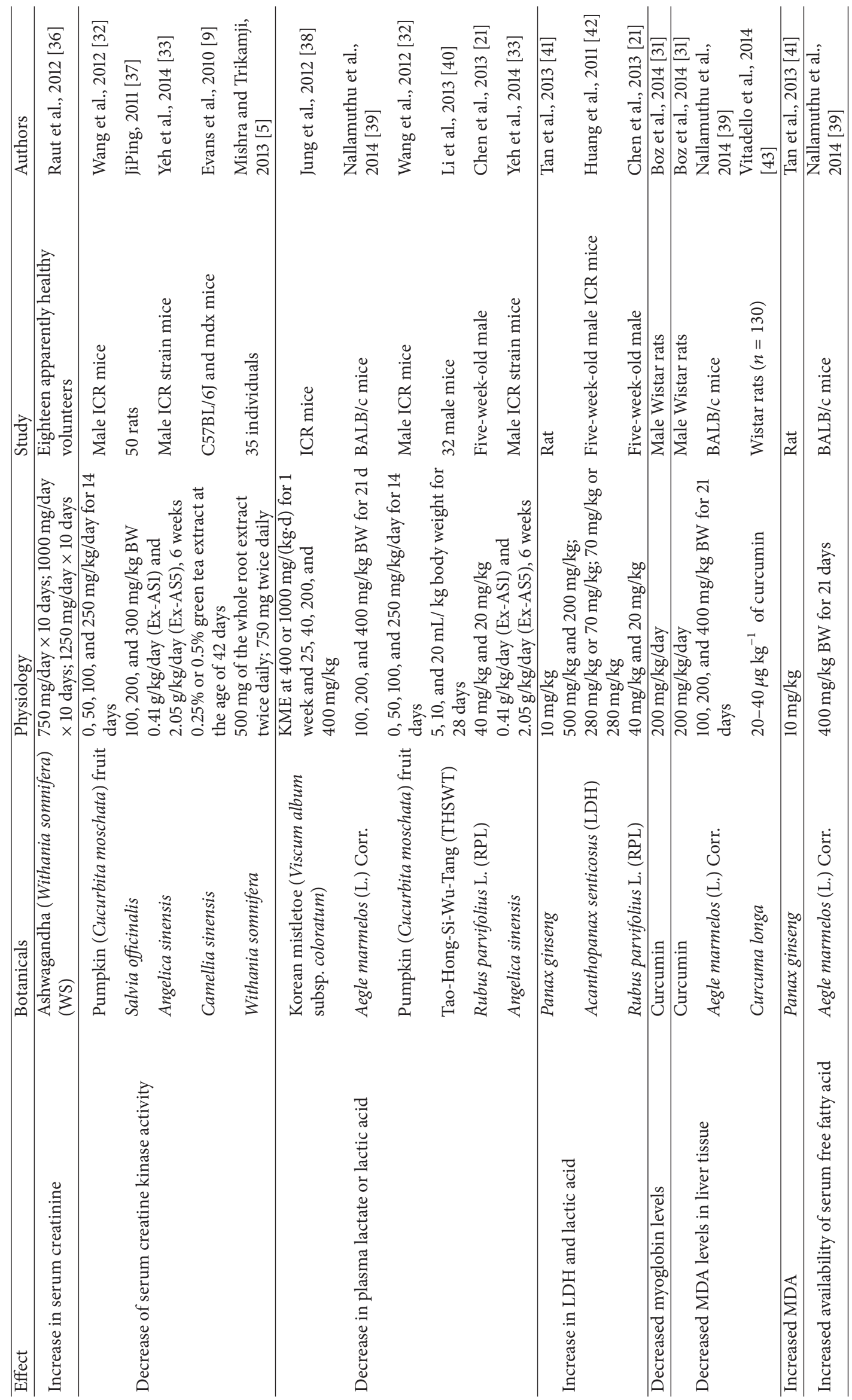




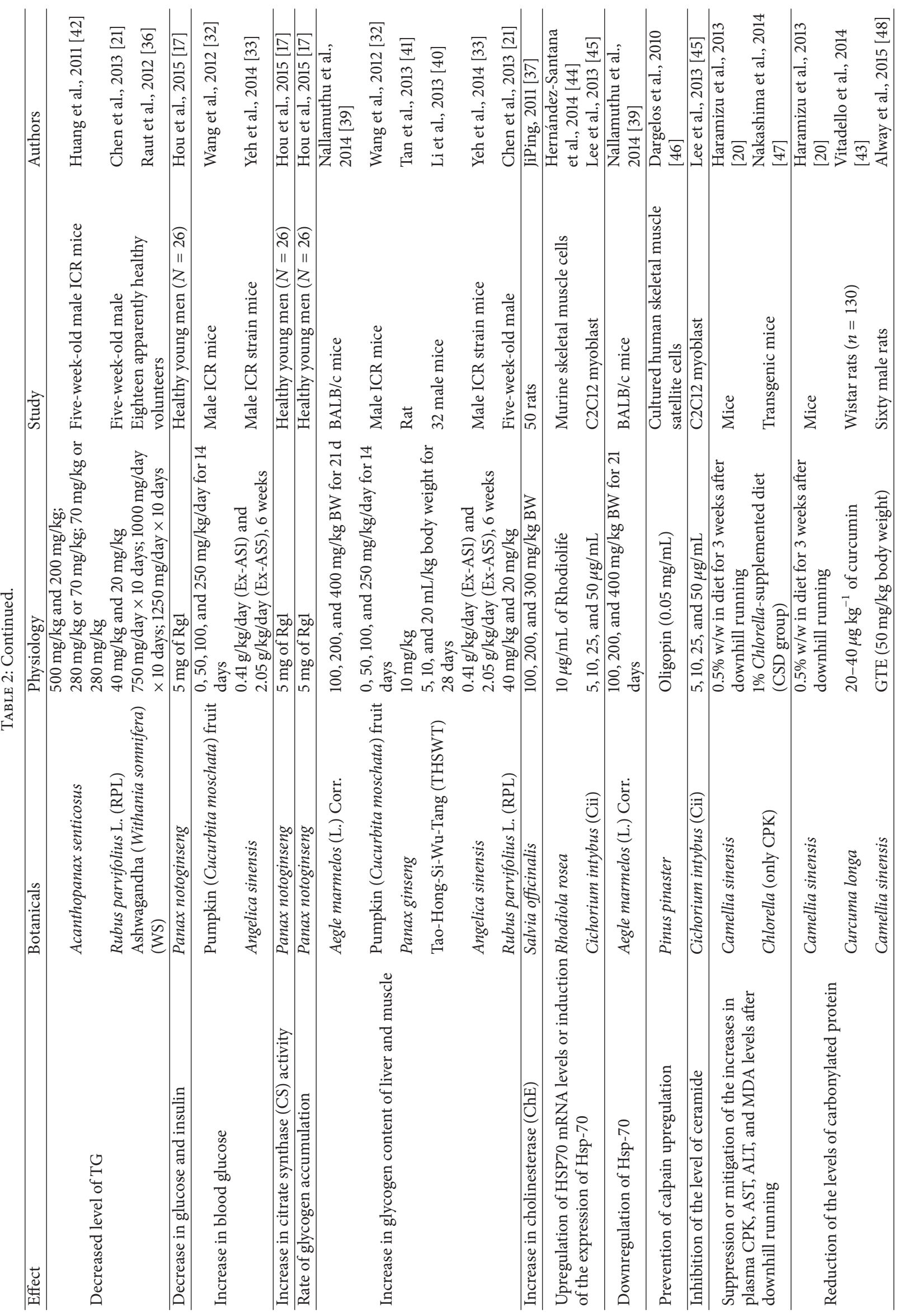




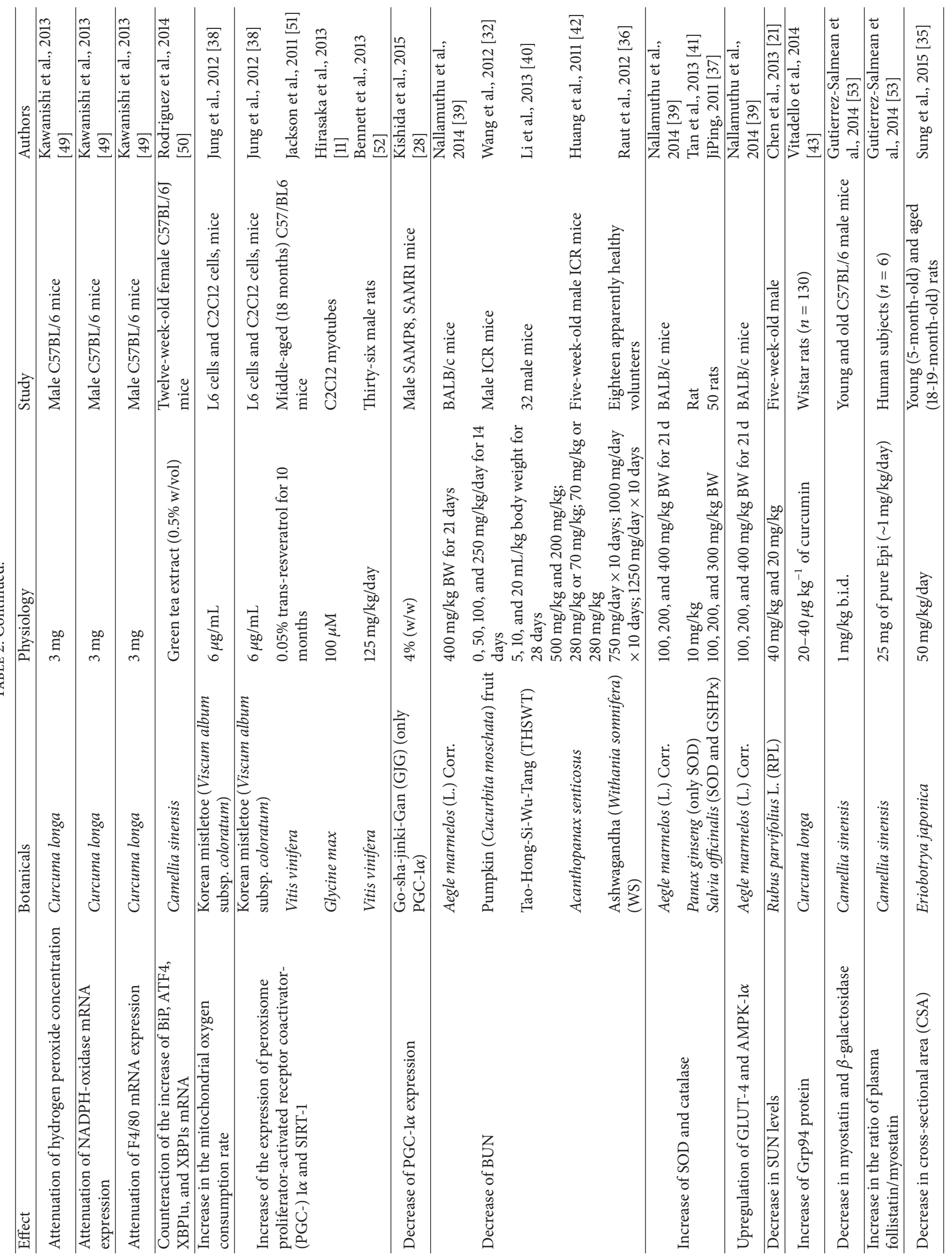




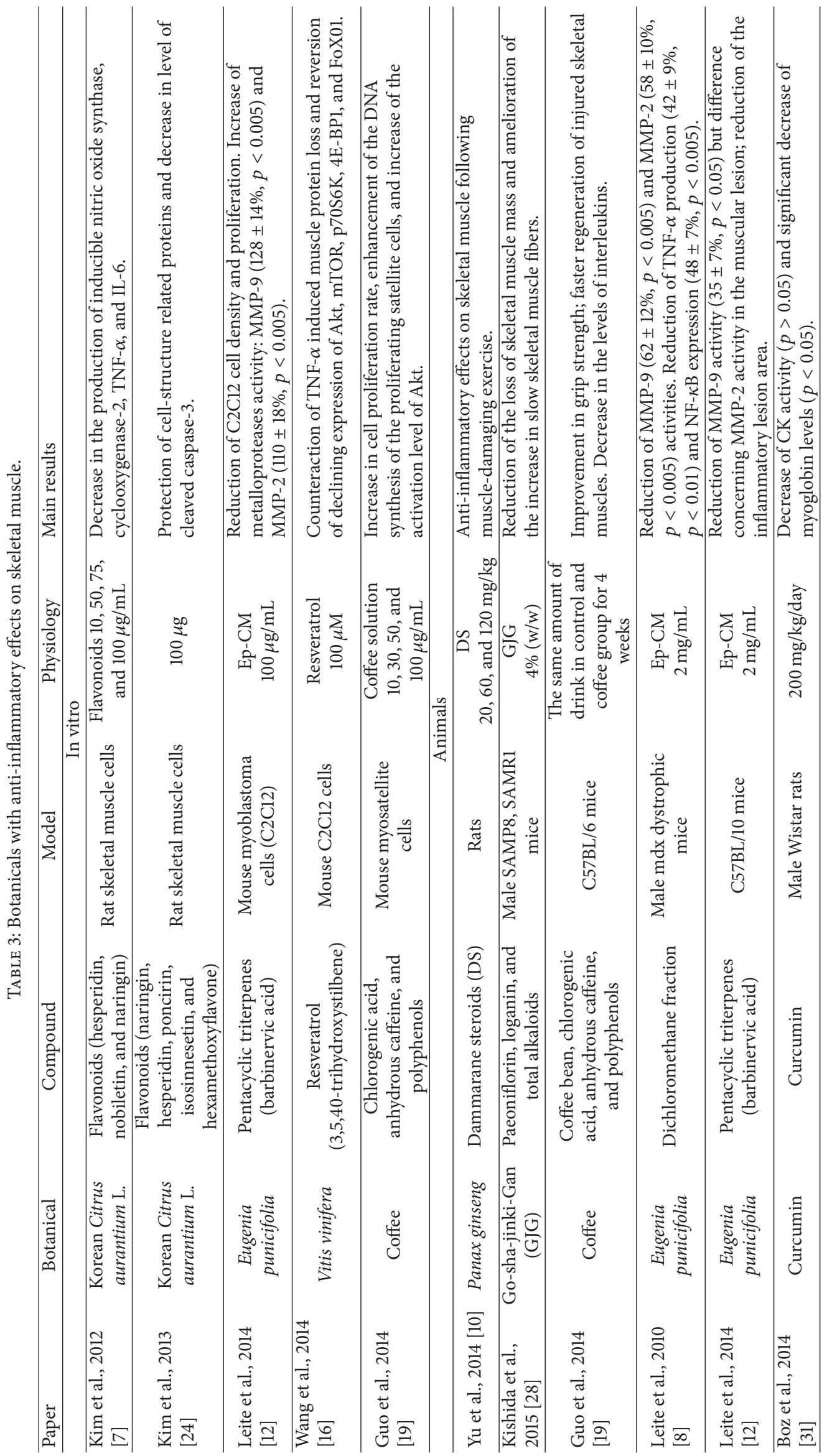




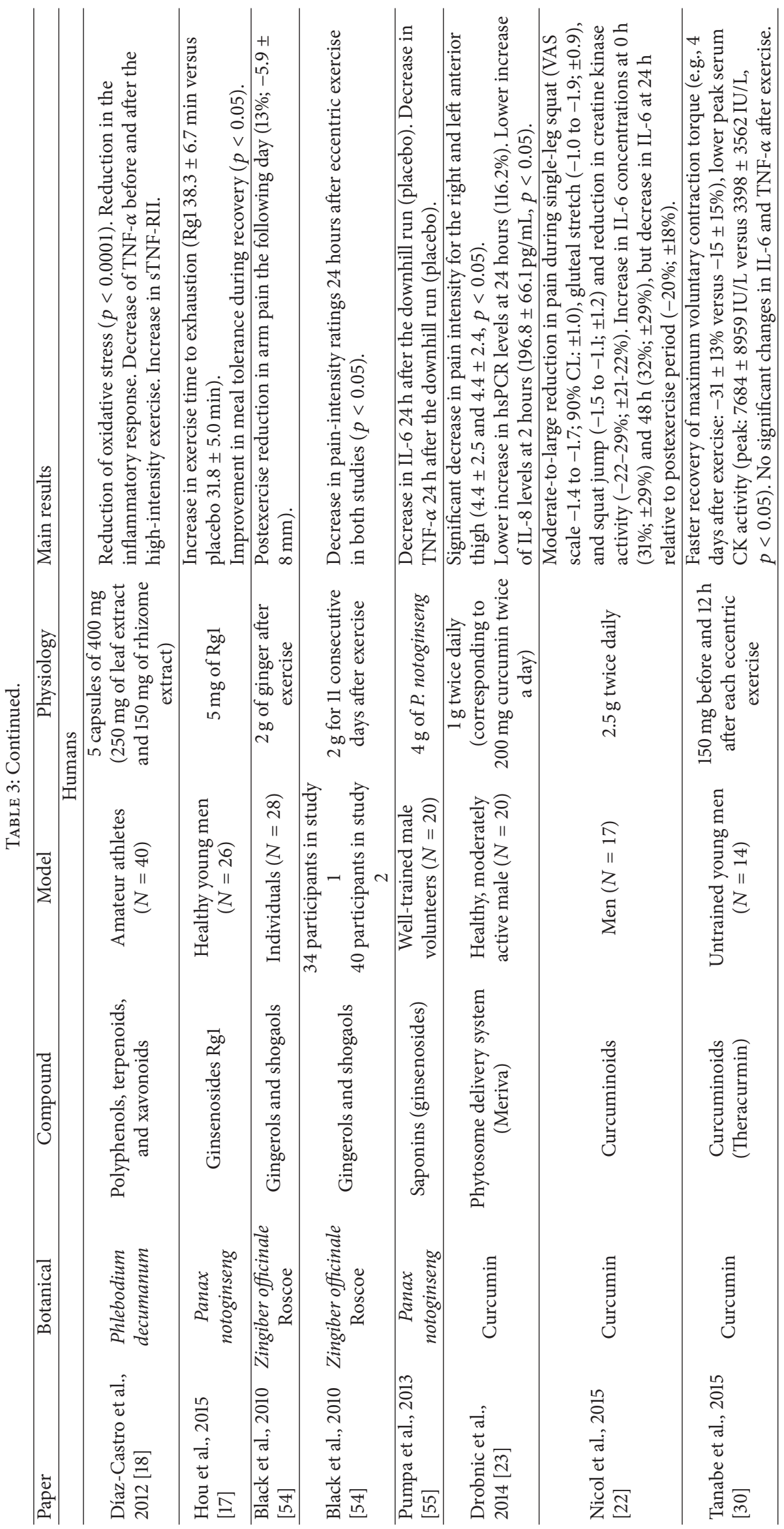




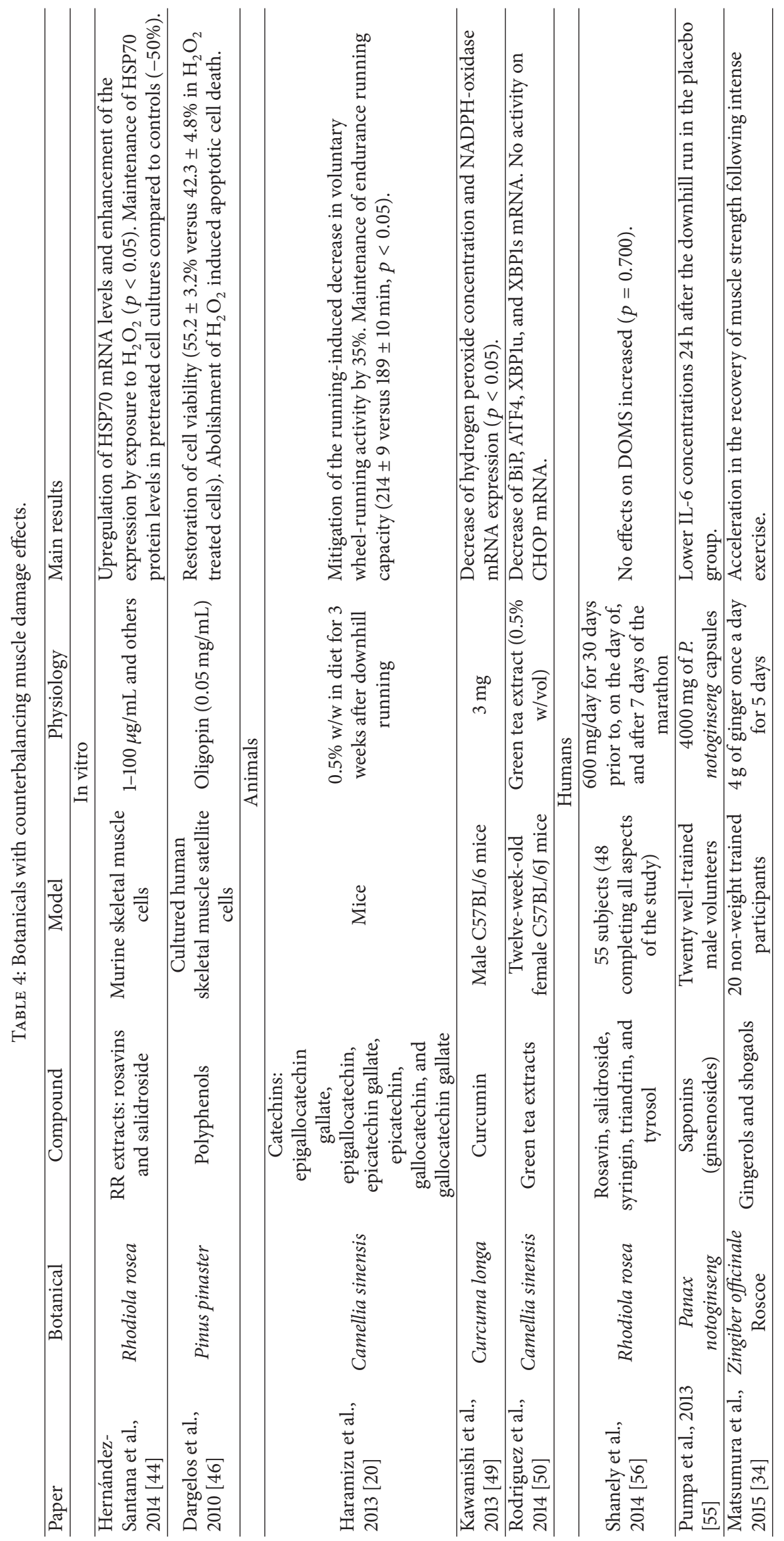




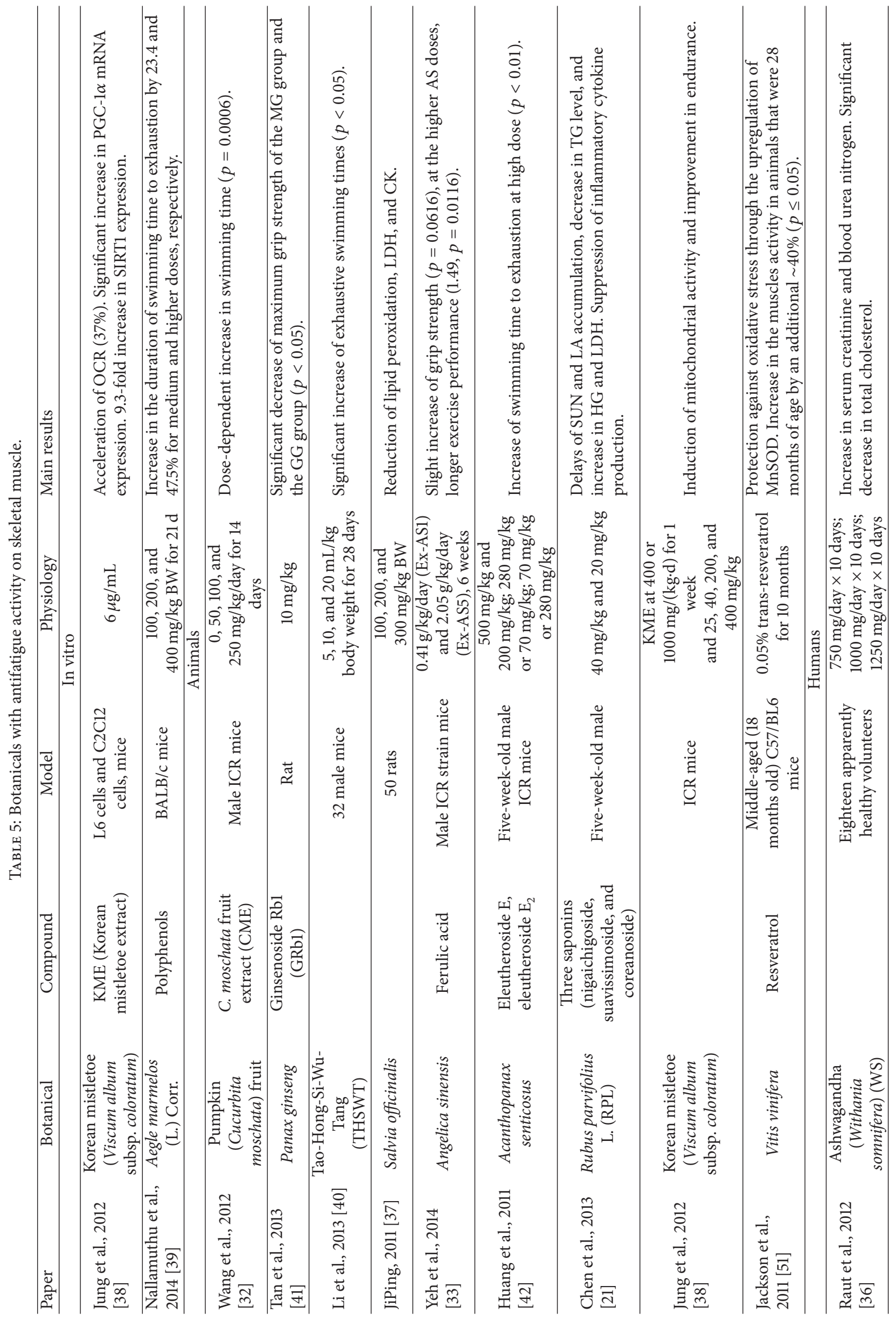




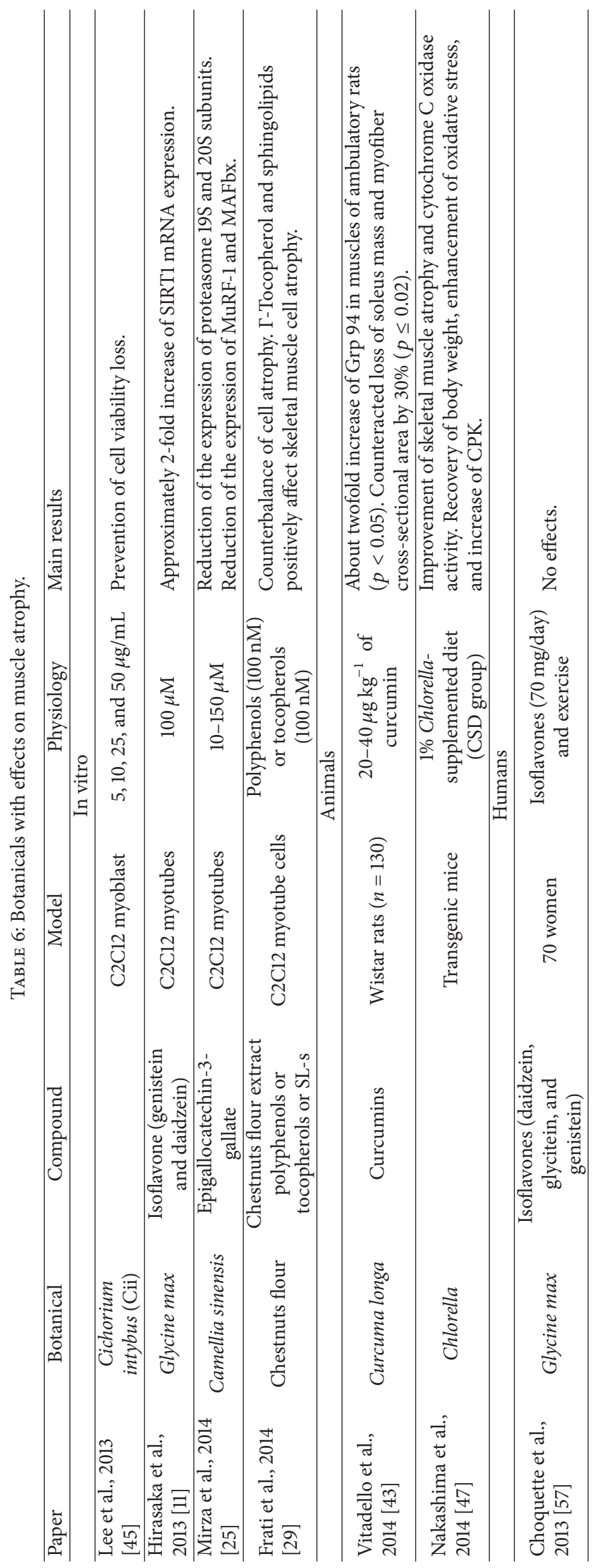


has no remarkable effect after single administration. In fact, only a moderate reduction in the progression of muscle pain from $24 \mathrm{~h}$ to $48 \mathrm{~h}$ following eccentric exercise was observed in participants who consumed ginger $24 \mathrm{~h}$ after exercise, and this effect was not enhanced by heat-treated ginger. In mice, Coffea decreases the levels of interleukins IL- $1 \alpha$ and IL- 6 and TNF- $\alpha$, which are correlated with muscle weight and grip strength. Using mice cells in vitro, coffee increases the number of proliferating cells and augmented DNA synthesis through the Akt signaling pathway. As a result, there is a combination of augmented satellite cell activation and decreased inflammatory levels by coffee treatment; it has anti-inflammatory effects both because it has antioxidant properties and because it has compounds, such as kahweol, with immunomodulatory properties [7, 19, 24, 54, 61]. Also, Eugenia punicifolia showed anti-inflammatory properties in the gastrocnemius muscle of mdx dystrophic mice; in particular, the activity of dichloromethane fraction of Eugenia punicifolia (Ep-CM), in mice, decreases metalloprotease- 9 and metalloprotease-2 activities (indicators of local inflammation and tissue remodeling, resp.) and levels of tumor necrosis factor- $\alpha$ and NF- $\kappa \mathrm{B}$ transcription factor [8]; isolated pentacyclic triterpene from Eugenia punicifolia reduces myoblast cells proliferation, has no effects on apoptosis, and increases matrix metalloproteases and muscular area (MMP9 and MMP-2) [12]. As shown in the study by Yu et al. [10], Dammarane steroids (DS) of Panax ginseng produce anti-inflammatory effects in rats, following muscle damage exercise, because they potentiate inflammation at baseline but exerted anti-inflammatory effects on skeletal muscle following muscle-damaging exercise. Another study has also highlighted the effect of steroid Rgl (capsule with $5 \mathrm{mg}$ of Rg1), an ergogenic component of ginseng, in healthy human against exercise challenge: the extract can minimize unwanted lipid peroxidation and attenuate proinflammatory shift under exercise challenge and so it ameliorates the postexercise recovery and mitochondria enzyme adaptation probably because the incorporation of the bulky steroid moiety of Rg1 into cellular membrane lipid may enhance molecular complexity and mechanical stability of the cell and mitochondrial membranes [17]. Panax notoginseng, as shown by Pumpa et al., seems to have no particular effects on interleukins, indicators of inflammation and muscle damage, in well-trained males after a bout of eccentric exercise designed to induce delayed-onset muscle soreness (DOMS) (in the experiment, $400 \mathrm{mg}$ of Panax notoginseng was used) [55]. Even Go-sha-jinki-Gan (GJG) maintains the area of muscle fibers in the soleus via normalizing signal transduction through the insulin-growth factor (IGF-1) Akt axis, the suppression of inflammation, and the maintenance of mitochondrial-related transcription factors in mice [28]. A positive effect on cell atrophy caused by TNF- $\alpha$ was shown with resveratrol (in Vitis vinifera) supplementation in a muscle cell line (regulating the Akt/mTOR/FoxO1 signaling pathways together with inhibition of the atrophy-related ubiquitin ligase) [16].

Finally, several studies have investigated the mechanisms by which curcumin, a constituent of turmeric (Curcuma longa L.), exerts its beneficial effect on muscle [62]. Early experimental study demonstrated that curcumin suppresses the activation of NF- $\kappa \mathrm{B}$, an effect of critical relevance in DOMS relief, since NF- $\kappa \mathrm{B}$ appears to be involved in the regulation of proteolysis and inflammation in muscle [62]. Therefore, inhibition of NF- $\kappa \mathrm{B}$ by curcumin may result in a muscle-protective effect. Consistently, it has been suggested that curcumin may prevent loss of muscle mass during sepsis and endotoxaemia and may stimulate muscle regeneration after traumatic injury [62]. Other mechanisms potentially responsible for the anti-inflammatory and antioxidant properties of curcumin include induction of heat-shock response [62], reduction in the expression of the proinflammatory enzyme cyclooxygenase-2 (COX-2), and promotion of the antioxidant response by activation of the transcription factor Nrf2 [63]. More recent studies confirm that curcumin can reduce inflammation and decrease some of the negative effects associated with eccentric exercise-induced muscle damage, including the release of proinflammatory cytokines and markers of muscle injury like creatine kinase (CK), as shown in animal models [31] and in in vitro settings [64].

The three studies that have been conducted until now in humans $[22,23,30]$ have shown that curcumin, at the dosages of $1 \mathrm{~g}$ twice daily (as the Phytosome ${ }^{\circledR}$ delivery system, Meriva ${ }^{\circledR}$ ) and $2.5 \mathrm{~g}$ twice daily, and $150 \mathrm{mg}$ of solid-lipid nanoparticle curcumin (Theracurmin ${ }^{\circledR}$ ), respectively, can prevent DOMS with some evidence of enhanced recovery of muscle performance, maximal voluntary contraction loss, and serum creatine kinase activity increase.

In conclusion, the muscle that makes activities undergoes an increase in inflammation that can damage the muscle itself. It is important to counteract the inflammatory activity in order to preserve the muscle from numerous types of damage. Several animal and in vitro studies have investigated the efficacy of botanicals with recognized antiinflammatory activity (such as Phlebodium decamanum, Citrus aurantium, Coffea arabica, Zingiber officinale, Eugenia punicifolia, Panax ginseng, Go-sha-jinki-Gan, Vitis vinifera, and Curcuma longa L.) on inflammation secondary to muscle activity (Table 2). These botanical extracts exerted their effects through different biochemical pathways, specifically decreasing interleukins or aging on transcriptional factors. Human studies were performed using four botanicals (Panax ginseng, Zingiber officinale, Phlebodium decumanum, and Curcuma longa L.) showing that (1) the daily consumption of raw and heat-treated Zingiber resulted in moderate-tolarge reductions in muscle pain after exercise-induced muscle injury; (2) Phlebodium supplementation for both professional and amateur athletes performing strenuous exercise resulted in reducing the undesirable effects of the oxidative stress and inflammation signaling elicited during high-intensity exercise; (3) Panax notoginseng did not convincingly have an effect on performance, muscular pain, or assessed blood markers in well-trained males after an intense bout of eccentric exercise that induced delayed-onset muscle soreness (DOMS); (4) curcumin could prevent DOMS enhancing the recovery of muscle performance and the maximal voluntary contraction loss and modulating the serum creatine kinase activity increase. 
All these clinical studies considered the reduction of inflammation and consequently muscle pain after a strenuous exercise and not in sarcopenic subjects, but this is a good starting point for the future utilization of these plants in the elderly.

3.3. Muscle Damage Prevention. This research has been carried out based on the keywords "skeletal muscle mass" and "damage" and "botanicals" or "plants" or "extracts"; 11 articles were sourced and 8 studies have been taken into consideration. Among these, 2 studies are in in vitro setting, 3 in animals, and 3 in humans (Table 4).

A recent study by Kawanishi et al. has clarified properties of curcumin after downhill running-induced muscle damage in mice. This study underlines how curcumin has an antioxidant effect in mice following downhill running-induced muscle damage; however, no differences in plasma creatine kinase $(\mathrm{CK})$ and plasma lactate dehydrogenase $(\mathrm{LDH})$, as markers of muscle damage, were observed. Curcumin administration immediately after downhill running did not prevent muscle damage but significantly attenuates the concentration of hydrogen peroxide and NADPH-oxidase gene expression; therefore, curcumin may be beneficial for the prevention of oxidative stress in downhill running-induced skeletal muscle damage [49]. Two recent studies in humans by Pumpa et al. [34] and Matsumura et al. [55] investigated the effects of Panax notoginseng (4000 mg) and Zingiber officinale (4 $\mathrm{g}$ for 5 days) on delayed-onset muscle soreness (DOMS); Zingiber officinale supplementation could have accelerated the recovery of maximal strength following muscle damage but did not prevent delayed muscle damage. The authors concluded that there is no evidence to support the use of Panax as a preventive option for DOMS and its related inflammation. Rhodiola rosea $(600 \mathrm{mg} / \mathrm{d})$ did not attenuate the postmarathon decrease in muscle function, the increases in muscle damage, the extracellular heatshock protein (eHSP72), or the plasma cytokines in human experienced runners [56]; however, the same plant modulates in vitro the expression of molecular factors (chaperone HSP70) such as heatshock proteins (HSP) in order to protect C2 C12 myotubes cells against peroxideinduced oxidative stress, suggesting a potential antioxidant role [44]. Finally, Haramizu et al. demonstrated that catechins of Camellia sinensis attenuate downhill running-induced muscle damage in mice, perhaps through their antioxidant properties, hastening recovery of physical performance [20]. A typical example of muscle damage is the cellular dysfunction caused by lipid excess. Lipid excess activates endoplasmatic reticulum (ER) stress in skeletal muscle and, as a consequence, accumulation of unfolded or misfolded proteins in ER lumen. Rodriguez et al. demonstrated that epigallocatechin-3-gallate (EGCG) from Camellia sinensis could protect mice muscle against ER stress, especially thanks to its antioxidant properties [50]. Dargelos et al. investigate the role of a natural antioxidant extracted from pine bark (Pinus pinaster) in cultured human skeletal muscle satellite cells. Results showed that this polyphenolic extract is able to protect cells from oxidative stress $\left(\mathrm{H}_{2} \mathrm{O}_{2}\right)$ damage and prevent the apoptosis and the activation of calpains mediated by $\mathrm{H}_{2} \mathrm{O}_{2}$ [46].
In conclusion (Table 3 ), until today, important studies were made on humans and animals for the prevention of muscle damage. Most of the plants used (Curcuma longa, Panax notoginseng, Zingiber officinale, Rhodiola rosea, Camellia sinensis, and Pinus pinaster) act on DOMS, thanks to their antioxidant properties. In human, Panax notoginseng seems to have no effect as a preventive option for DOMS, and Rhodiola rosea does not attenuate muscle damage. Further studies are needed but we can say that botanical supplementation, thanks to its antioxidant properties, could be useful to prevent sarcopenia due to the fact that the loss of muscle mass in aging is driven also by oxidative stress, as it happens after strenuous exercise.

3.4. Antifatigue. This research has been carried out based on the keywords "skeletal muscle mass" and "fatigue" and "botanicals" or "plants" or "extracts"; 20 articles were sourced and 11 studies are taken into account. Among these, only one study is made in humans, one in in vitro settings, and one both in in vitro settings and in animals and the others are made in animals (Table 5).

Tan et al. in 2013 investigated for the first time the role of ginsenoside Rb1 (Grb1) in Panax quinquefolius, as antifatigue agent, on postoperative fatigue syndrome (POFS) in a rat model induced by major small intestinal resection, through its antioxidant properties and the improvement of energy metabolism. Grb1 enhances maximum grip strength and increases the activity of lactate dehydrogenase and other biochemical parameters. The results suggested that GRb1 improves the maintenance of normal $\mathrm{pH}$ range in muscle tissue by reducing the accumulation of lactic acid (LA) and attenuates LA induced side effects of various biochemical and physiological processes, which impair bodily performance [41]. In accordance, the study by Nallamuthu et al. demonstrated the antifatigue properties in mice of A. marmelos fruit, most probably manifested by delaying the accumulation of serum lactic acid, increasing the fat utilization, and upregulating the skeletal muscle metabolic regulators [39]. Likewise, Salvia sativa, Angelica sinensis, Cucurbita moschata, Withania somnifera, and Acanthopanax senticosus extracts exhibit different antifatigue effects. All of these studies, with the exception of Withania somnifera, are performed in animals (rats or mice). These studies demonstrate that the antioxidant properties of plants play an important role in reducing fatigue. Salvia reduces lipid peroxidation, lactate dehydrogenase, creatine kinase activities, enhanced antioxidant enzymes, and cholinesterase (ChE) activities in the skeletal muscle of endurance exercise rats; similar effects have been observed for other extracts, with some differences between each other, in which, additionally, antifatigue is measured also by forelimb grip strength and exhaustive swimming time as well as serum levels of lactate, ammonia, glucose, and creatine kinase after a 15 min swimming exercise. Specifically, the mechanisms of Acanthopanax (also called Eleutherococcus senticosus or Siberian ginseng) are the reduction of the level of triglycerides by increasing fat utilization, the delay of the accumulation of blood urea nitrogen (BUN), and the increase of the lactate dehydrogenase $(\mathrm{LDH})$ to reduce the accumulation of lactic acid in muscle 
and then protect the muscle tissue [32, 33, 36, 37, 42]. Strange but active is Tao-Hong-Si-Wu-Tang that shows antifatigue activity in mice due to extended exhaustive swimming time, the increase of liver and muscle glycogen contents, and the decrease of the lactic acid (BLA) and urea nitrogen (BUN) plasmatic contents [40]. Also, Chen et al. define the antifatigue property of Rubus parvifolius L. (RPL) in experiment with mice, finding that total saponins from RPL possess potent capabilities to alleviate fatigue induced by forced swimming and that nigaichigoside F1 was responsible for the pharmacological effect. The underlying mechanisms include delays in the accumulation of serum urea nitrogen (SUN) and lactic acid (LA), a decrease in TG level by increasing fat consumption, increases in hepatic glycogen (HG) and LDH so that lactic acid accumulation was decreased, the reduction of ammonia in the muscle, and the suppression of increased immune activation and inflammatory cytokine production [21]. Viscum album subsp. coloratum increase mitochondrial oxygen consumption rate (OCR) in L6 cells and increase the expression of peroxisome proliferator-activated receptor c coactivator- (PGC-) la and silent mating type information regulation 2 homolog 1 (SIRT1), two major regulators of mitochondria function, in $\mathrm{C} 2 \mathrm{C} 12$ cells, suggesting that this extract has great potential as a novel mitochondria-activating agent and could exert the antifatigue effect [38]. Jackson et al. try to understand how Vitis vinifera and its compound resveratrol could prevent muscle fatigue. Resveratrol has a protective effect against aging-induced oxidative stress in skeletal muscle, likely through the upregulation of manganese superoxide dismutase (MnSOD) activity, reducing hydrogen peroxide, and lipid peroxidation levels in muscle samples, but sarcopenia was not attenuated by resveratrol [51].

Withania somnifera (gradual escalating doses from 750 to $1250 \mathrm{mg} /$ day) in humans has demonstrated muscle strengthening and lipid lowering [36].

In conclusion, there are several preclinical lines of evidence that botanical extracts, such as Panax quinquefolius, $A$. marmelos fruit, Salvia sativa, Angelica sinensis, Phalaenopsis cornu-cervi, Cucurbita moschata, Withania somnifera, Acanthopanax senticosus, deer antler extract, Tao-Hong-Si-WuTang, Rubus parvifolius L., velvet antler extract, Viscum album subsp. coloratum, and Vitis vinifera, can reduce the muscle's fatigue, after intense exercise or simply in a condition of loss of muscle mass, as in sarcopenia (Table 4). Commonly, these properties are due to their antioxidant effects: in general, these plants reduce lipid peroxidation, lactic acid, and serum levels of ammonia and creatine kinase and increase liver and muscle glycogen. The only study found in human was that of Raut et al., in which supplementation with Withania somnifera (with gradual escalating doses from 750 to $1250 \mathrm{mg} /$ day) seems to have good effects on antifatigue, but this is a preliminary study. Until today, the role of plants in antifatigue in clinical studies has been not deeply documented and so it is difficult to recommend particular supplementation.

3.5. Muscle Atrophy Prevention. This research has been carried out based on the keywords "skeletal muscle mass" and "atrophy" and "botanicals" or "plants" or "extracts"; 15 articles were sourced and 7 studies are taken into account. Among these, 4 are in in vitro settings and 2 are in animals and only one is in human (Table 6).

Curcuma longa can prevent muscle atrophy. It stimulates glucose-regulated protein $94 \mathrm{kDa}$ (Grp94) expression in myogenic cells, whose levels decrease significantly in unloaded muscle, and it is involved in attenuation of myofiber atrophy in rats [43]. Also, Camellia sinensis extracts in rats appear to counteract the increased protein degradation (linked with its ability to downregulate key components of the ubiquitin proteasome proteolytic pathway) [25]. Instead, Cichorium intybus extract prevents skeletal muscle atrophy in vitro, probably increasing heat-shock protein-70 (Hsp-70) production and inhibiting the level of ceramide: Hsp-70, in fact, has a positive effect on reducing oxidative stress of cells and ceramide is involved in the regulation of cell death [45]. Also, chestnut sweet flour (rich in $\gamma$-tocopherol) protects from skeletal muscle cell atrophy, but this protection appears not to be due to a general antioxidant action, but maintaining cellular redox homeostasis through the regulation of NADPH oxidase, mitochondrial integrity [29]. Isoflavones are the most important phytochemicals in Glycine max for preventing muscle atrophy. These products could induce in vitro the expression of SIRT-1, a sirtuin that normally deacetylates p65, in order to reduce the activity of MuRF-1 related to muscle atrophy. Overall, they suppress MuRF-1 promoter activity and myotube atrophy induced by TNF- $\alpha$ in $\mathrm{C} 2 \mathrm{C} 12$ myotubes [11]. However, a study performed by Choquette et al. demonstrated that, in postmenopausal women, only exercise, but not soy isoflavones $(70 \mathrm{mg} /$ day), could improve muscle strength and reduce risks of mobility impairments [57]. In addition, consumption of Chlorella, a unicellular green alga, could prevent age-related muscle atrophy in mice, because it contains various antioxidant substances, including carotenoids and vitamins and plastoquinone that has been shown to hold greater antioxidant properties. Chlorella contains also amino acids such as the brain chain amino acids (BCAA) valine, leucine, and isoleucine, which are important components of actin and myosin, the fundamental muscle proteins, and may be important in prevention of sarcopenia. Finally, Chlorella also prevents mitochondrial dysfunction [47].

In conclusion, it is clear that botanical extracts can prevent the atrophy of muscle, after intense exercise or simply in a condition of loss of muscle mass, as in sarcopenia. We considered several botanicals (Curcuma longa, Camellia sinensis, Cichorium intybus, chestnut sweet flour, Glycine max, and Chlorella): most of them have important antioxidant properties, which prevent muscle's atrophy. However, the only study made on human, using Glycine max, did not show positive results and so other researches are needed to substantiate the use of botanicals supplementation to prevent muscle atrophy.

3.6. Muscle Regeneration and Differentiation. This research has been carried out based on the keywords "skeletal muscle mass" and "regeneration" and "botanicals" or "plants" or "extracts"; 19 articles were sourced and 15 studies are taken into account. Among these, 4 are in in vitro settings, 7 in 
animals, and 3 in human and one is both in animals and in humans (Table 7). Nutraceutical compounds by $C$. sinensis in mice decrease myostatin and $\beta$-galactosidase and increase levels of markers of muscle; instead, in humans, they (7-day treatment with epicatechin at $1 \mathrm{mg} / \mathrm{kg} /$ day) increase hand grip strength and the ratio of plasma follistatin/myostatin [53] and regulate NF- $\kappa \mathrm{B}$ activity in regenerating muscle fibers [9]. Camellia also induces changes in satellite cell number and it improves muscle recovery following a period of atrophy in old rats and decreases oxidative stress, but this is insufficient to improve muscle recovery following a period of atrophy [48]. Also, an increase in myogenin (due to a supplement of Vitis vinifera resveratrol extracts) served to stimulate differentiation to compensate for an impaired function of satellite cells (SCs) in the old muscles [26]. An article by Ballak et al., about resveratrol, says that this compound does not rescue the hypertrophic response and even reduces the number of satellite cells in hypertrophied muscle of mice [26]. Also, Ferula hermonis Boiss. and Vitis vinifera significantly increase muscle weight and enhance the growth of skeletal muscle fibers or fiber size (increase the fiber cross-sectional area of type IIA and IIB fibers) and nuclear number in order to enhance the growth of skeletal muscle [52, 58]. It is noteworthy that proanthocyanidins of Vitis have been used in a clinical trial. An increase of muscle mass and the improvement of several physical conditions have been observed in middle-aged women (with at least one menopausal symptom) treated with doses from 100 to $200 \mathrm{mg} / \mathrm{d}$ [60]. Broussonetia kazinoki (B. kazinoki), Corydalis turtschaninovii, and Hachimijiogan, in vitro, promote myogenic differentiation through activation of key promyogenic kinase (p38 MAPK) or ERK1/2 and MyoD transcription activities (MyoD family transcription factors play a key role in promoting myoblast differentiation) without affecting the Akt signaling pathway [13-15]. Another in vitro study, performed by Poussard et al., indicated Oligopin, a pine bark extract, as natural antioxidant; in fact, with aging, oxidative stress produces disruption of cytoskeleton and phosphorylated heat-shock protein beta-1 (HSPB1) may help to repair injured structures. Furthermore, Oligopin prevents the stress-induced phosphorylation of HSPB-1 in human cells [27]. Curcumin (Curcuma longa) may modulate the entry into apoptosis during immobilization and stimulate initial steps of muscle regeneration, aging on proteins and enzyme such as proteasome chymotrypsin-like activity and proapoptotic smac/DIABLO protein levels, and apoptosome-linked caspase-9 activities [59]. Another study was performed in humans with Withania somnifera: it seems to improve muscle strength and endurance for the aged subjects and so it could be used in preventing sarcopenia (500-750 mg twice daily for three months) [5]. Finally, Kim et al. demonstrate that physical exercise combined with tea catechin supplementation $(350 \mathrm{~mL}$ of a tea beverage fortified with $540 \mathrm{mg}$ of catechins) had a beneficial effect on physical function measured by walking ability and muscle mass in women with sarcopenia [65].

Lastly, a very recent study [35] demonstrated in animal models that loquat (Eriobotrya japonica) leaf extract (LE) diminished the age-associated loss of grip strength and enhanced muscle mass and muscle creatine kinase
(CK) activity. Histochemical analysis revealed that loquat (Eriobotrya japonica) leaf extract (LE) abrogated the ageassociated decrease in cross-sectional area (CSA) and decreased the amount of connective tissue in the muscle of aged rats. Moreover, in order to investigate the mode of action, $\mathrm{C} 2 \mathrm{C} 12$ murine myoblasts were used to evaluate the myogenic potential of LE. The expression levels of myogenic proteins (MyoD and myogenin) and functional myosin heavy chain (MyHC) were measured by western blot analysis. LE enhanced MyoD, myogenin, and MyHC expression. The changes in the expression of myogenic genes corresponded to an increase in the activity of CK, a myogenic differentiation marker. Finally, loquat (Eriobotrya japonica) leaf extract (LE) activated the Akt/mammalian target of rapamycin (mTOR) signaling pathway, which is involved in muscle protein synthesis during myogenesis. These findings suggest that loquat (Eriobotrya japonica) leaf extract (LE) attenuates sarcopenia by promoting myogenic differentiation and subsequently promoting muscle protein synthesis.

In conclusion, there are several preclinical lines of evidence for a variety of plants (Camellia sinensis, Vitis vinifera, Ferula hermonis Boiss., grape seed, Broussonetia kazinoki, Corydalis turtschaninovii, Hachimijiogan, pine bark, Curcuma longa, Withania somnifera, and Eriobotrya japonica), but only four studies are available in humans: two of these were conducted with supplementation of Camellia sinensis products, one with Withania somnifera and one with grape seed. In particular, the use of Withania somnifera (50-750 mg twice a day) resulted in improving muscle strength in human and also the supplementation with $540 \mathrm{mg}$ of catechin from Camellia sinensis induced positive physical improvement. The second study demonstrated an improvement in grip strength, but it was only an experimental study with $25 \mathrm{mg}$ of pure EGCG. Finally, the clinical trial with grape seed (100-200 mg/d) seemed to increase muscle mass and improve other physical conditions during menopause. For muscle regeneration, the main studies to take into account were those performed by Kim et al. and by Mishra et al., in which sarcopenic subjects have been enrolled. However, it is clear that the supplementation with EGCG should be complementary to appropriate physical exercise in order to reach the beneficial effects on muscle mass and that further studies are needed also for Withania supplementation.

\section{Discussion}

Currently, only diet and exercise are recognized as an effective means to counteract loss of muscle [53]. Regarding exercise, it is important to note that exercise-induced muscle damage (EIMD) can be caused by eccentric type or unaccustomed (novel) exercise and results in decrements in muscle force production, development of delayed-onset muscle soreness (DOMS) and swelling, rise in passive tension, and an increase in blood intramuscular proteins [66].

Delayed-onset muscle soreness is generally considered a hallmark sign of EIMD [67], and it is thought that DOMS is partially related to direct muscle fiber damage, and its magnitude appears to vary with the type, duration, and intensity of exercise [68]. 


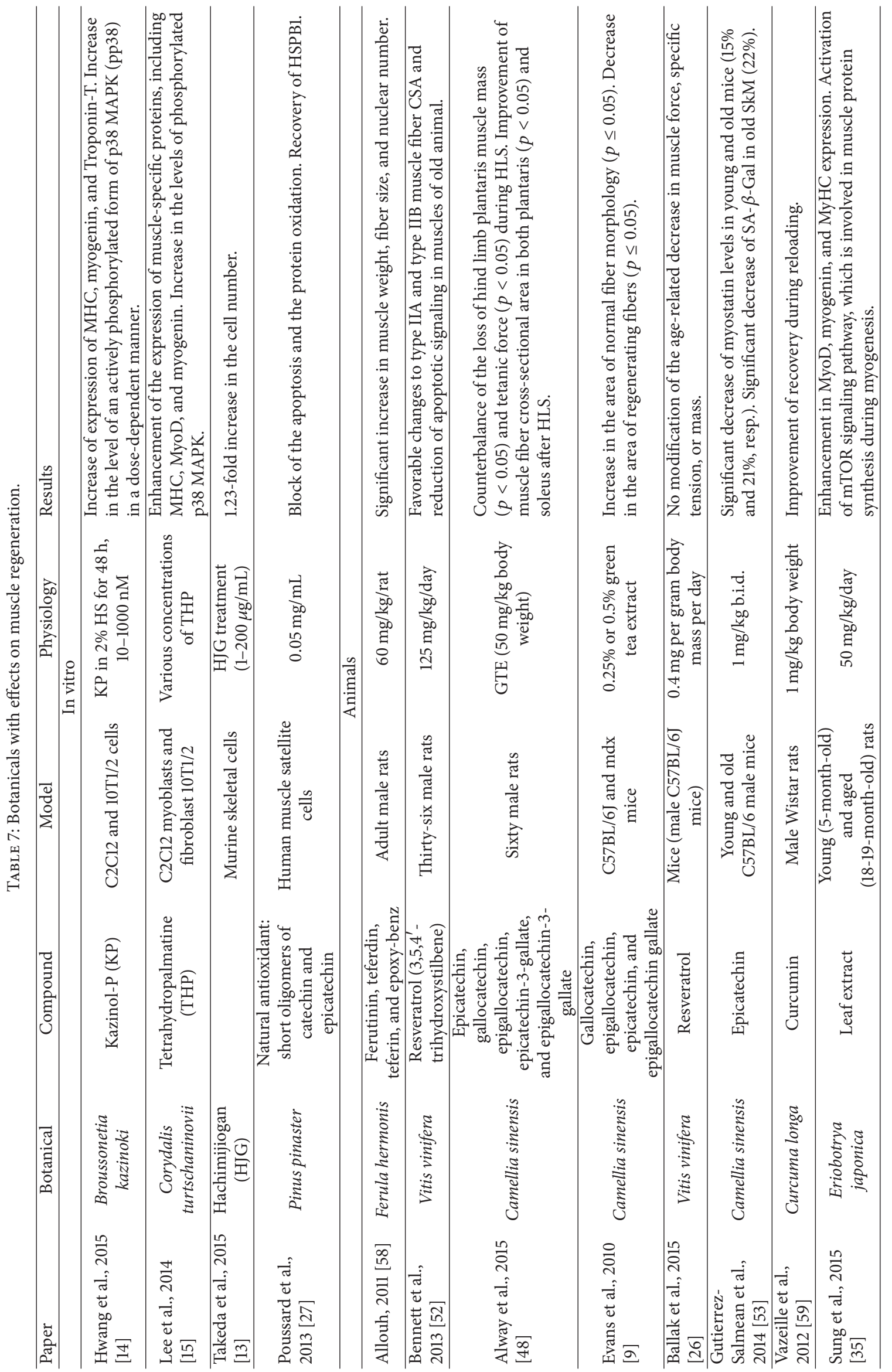




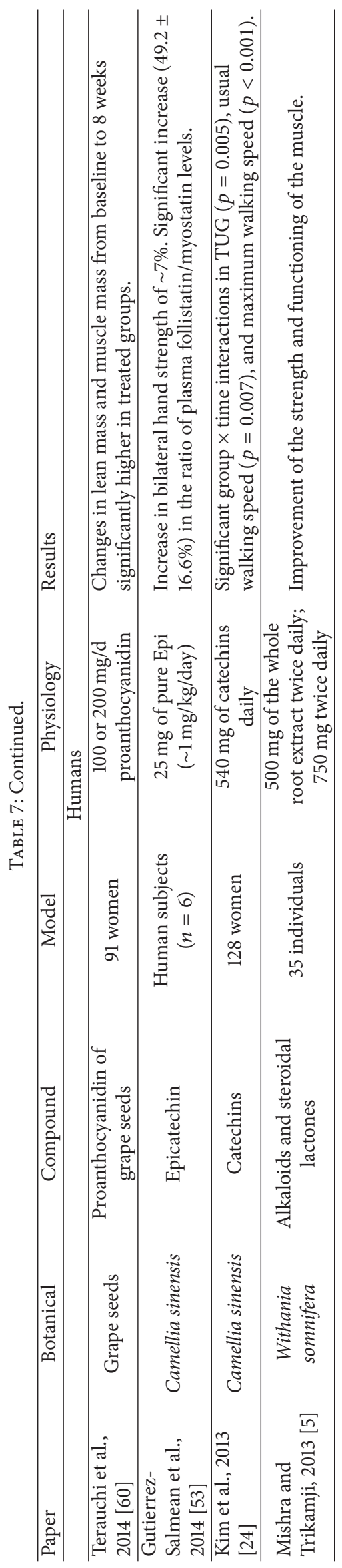


The inflammatory response to EIMD results in the release into blood of reactive species from both neutrophils and macrophages and an array of cytokines from the injured muscle including tumor necrosis factor- (TNF-) $\alpha$, interleukin(IL-) $1 \beta$, and IL-6, which contribute to low-grade systemic inflammation and oxidative stress [69]. The proinflammatory and prooxidant response can provoke secondary tissue damage [70], thus prolonging the regenerative process, which is generally characterized by restoration of muscle strength and resolution of inflammation [70]. All these phenomena must be avoided in elderly sarcopenic subjects and so it is critical in this population to better preserve skeletal muscle and muscle function.

In this review, we focused our attention on effects of several botanicals on growth and health of muscle and we divided these effects into five categories: anti-inflammation, muscle damage prevention, antifatigue, muscle atrophy prevention, and muscle regeneration and differentiation.

To date, although the animal studies and in vitro studies are numerous and promising, studies in humans evaluating the effectiveness of anti-inflammatory and antioxidant activities of botanicals on welfare of skeletal muscle are still very few.

Although only relatively few human studies have been published on the potential use of botanicals for the prevention and treatment of muscle function, the present review is important because it highlights the need of continued efforts to find effective treatment of this debilitating condition. The available results, in particular considering human studies, suggest that the botanicals that may be potentially useful dietary supplements to prevent loss of muscle mass and function are curcumin from Curcuma longa, alkaloids and steroidal lactones from Withania somnifera (Solanaceae), catechins from Camellia sinensis, proanthocyanidin of grape seeds, and gingerols and shogaols from Zingiber officinale.

It should be noted that this review is not claiming that the use of these botanicals has been proven to prevent and treat loss of muscle mass and muscle function, but we believe that early and preliminary observations are promising. Further researches will support the use of these botanicals in the management of age-related muscle dysfunction and this may open the possibility of treating age-related loss of muscle mass and function with supplements.

\section{Conflict of Interests}

The authors declare no conflict of interests regarding the publication of this paper.

\section{References}

[1] Y. Rolland, S. Czerwinski, G. Abellan Van Kan et al., "Sarcopenia: its assessment, etiology, pathogenesis, consequences and future perspectives," The Journal of Nutrition Health and Aging, vol. 12, no. 7, pp. 433-450, 2008.

[2] A. J. Cruz-Jentoft, J. P. Baeyens, J. M. Bauer et al., "European working group on sarcopenia in older people. Sarcopenia: European consensus on definition and diagnosis: report of the European working group on sarcopenia in older people," Age and Ageing, vol. 39, no. 4, pp. 412-423, 2010.
[3] R. A. McGregor, D. Cameron-Smith, and S. D. Poppitt, "It is not just muscle mass: a review of muscle quality, composition and metabolism during ageing as determinants of muscle function and mobility in later life," Longevity \& Healthspan, vol. 3, no. 1, article 9, 2014.

[4] M. Rondanelli, M. Faliva, F. Monteferrario et al., "Novel insights on nutrient management of sarcopenia in elderly," BioMed Research International, vol. 2015, Article ID 524948, 14 pages, 2015.

[5] S. K. Mishra and B. Trikamji, "A clinical trial with Withania somnifera (Solanaceae) extract in the management of sarcopenia," Signpost Open Access Journal of Organic and Biomolecular Chemistry, vol. 1, pp. 187-194, 2013.

[6] M. Egger, K. Dickersin, and G. D. Smith, "Problems and limitations in conducting systematic reviews," in Systematic Reviews in Health Care: Meta-Analysis in Context, M. Egger, G. D. Smith and, and D. G. altman, Eds., chapter 3, BMJ Books, London, UK, 2nd edition, 2001.

[7] J.-A. Kim, H.-S. Park, S.-R. Kang et al., "Suppressive effect of flavonoids from Korean citrus aurantium L. on the expression of inflammatory mediators in L6 skeletal muscle cells," Phytotherapy Research, vol. 26, no. 12, pp. 1904-1912, 2012.

[8] P. E. C. Leite, K. B. de Almeida, J. Lagrota-Candido et al., "Anti-inflammatory activity of Eugenia punicifolia extract on muscular lesion of mdx dystrophic mice," Journal of Cellular Biochemistry, vol. 111, no. 6, pp. 1652-1660, 2010.

[9] N. P. Evans, J. A. Call, J. Bassaganya-Riera, J. L. Robertson, and R. W. Grange, "Green tea extract decreases muscle pathology and NF- $\kappa \mathrm{B}$ immunostaining in regenerating muscle fibers of mdx mice," Clinical Nutrition, vol. 29, no. 3, pp. 391-398, 2010.

[10] S.-H. Yu, C.-Y. Huang, S.-D. Lee et al., "Decreased eccentric exercise-induced macrophage infiltration in skeletal muscle after supplementation with a class of ginseng-derived steroids," PLoS ONE, vol. 9, no. 12, Article ID el14649, 2014.

[11] K. Hirasaka, T. Maeda, C. Ikeda et al., "Isoflavones derived from soy beans prevent MuRF1-mediated muscle atrophy in $\mathrm{C} 2 \mathrm{C} 12$ myotubes through SIRT1 activation," Journal of Nutritional Science and Vitaminology, vol. 59, no. 4, pp. 317-324, 2013.

[12] P. E. Leite, K. G. Lima-Araújo, G. R. França, J. Lagrota-Candido, W. C. Santos, and T. Quirico-Santos, "Implant of polymer containing pentacyclic triterpenes from Eugenia punicifolia inhibits inflammation and activates skeletal muscle remodeling," Archivum Immunologiae et Therapiae Experimentalis, vol. 62, no. 6, pp. 483-491, 2014.

[13] T. Takeda, K. Tsuiji, B. Li, M. Tadakawa, and N. Yaegashi, "Proliferative effect of Hachimijiogan, a Japanese herbal medicine, in $\mathrm{C} 2 \mathrm{C} 12$ skeletal muscle cells," Clinical Interventions in Aging, vol. 10, pp. 445-451, 2015.

[14] J. Hwang, S.-J. Lee, M. Yoo et al., "Kazinol-P from Broussonetia kazinoki enhances skeletal muscle differentiation via p38MAPK and MyoD," Biochemical and Biophysical Research Communications, vol. 456, no. 1, pp. 471-475, 2015.

[15] S.-J. Lee, M. Yoo, G.-Y. Go et al., “Tetrahydropalmatine promotes myoblast differentiation through activation of p38MAPK and MyoD," Biochemical and Biophysical Research Communications, vol. 455, no. 3-4, pp. 147-152, 2014.

[16] D.-T. Wang, Y. Yin, Y.-J. Yang et al., "Resveratrol prevents TNF$\alpha$-induced muscle atrophy via regulation of Akt/mTOR/FoxO1 signaling in C2C12 myotubes," International Immunopharmacology, vol. 19, no. 2, pp. 206-213, 2014. 
[17] C.-W. Hou, S.-D. Lee, C.-L. Kao et al., "Improved inflammatory balance of human skeletal muscle during exercise after supplementations of the ginseng-based steroid rgl," PLOS ONE, vol. 10, no. 1, Article ID e0116387, 2015.

[18] J. Díaz-Castro, R. Guisado, N. Kajarabille et al., "Phlebodium decumanum is a natural supplement that ameliorates the oxidative stress and inflammatory signalling induced by strenuous exercise in adult humans," European Journal of Applied Physiology, vol. 112, no. 8, pp. 3119-3128, 2012.

[19] Y. Guo, K. Niu, T. Okazaki et al., "Coffee treatment prevents the progression of sarcopenia in aged mice in vivo and in vitro," Experimental Gerontology, vol. 50, no. 1, pp. 1-8, 2014.

[20] S. Haramizu, N. Ota, T. Hase, and T. Murase, "Catechins suppress muscle inflammation and hasten performance recovery after exercise," Medicine and Science in Sports and Exercise, vol. 45, no. 9, pp. 1694-1702, 2013.

[21] J. Chen, X. Wang, Y. Cai et al., "Bioactivity-guided fractionation of physical fatigue-attenuating components from Rubus parvifolius L," Molecules, vol. 18, no. 9, pp. 11624-11638, 2013.

[22] L. M. Nicol, D. S. Rowlands, R. Fazakerly, and J. Kellett, "Curcumin supplementation likely attenuates delayed onset muscle soreness (DOMS)," European Journal of Applied Physiology, vol. 115, no. 8, pp. 1769-1777, 2015.

[23] F. Drobnic, J. Riera, G. Appendino et al., "Reduction of delayed onset muscle soreness by a novel curcumin delivery system $\left(\right.$ Meriva $\left.^{5}\right)$ : a randomised, placebo-controlled trial," Journal of the International Society of Sports Nutrition, vol. 11, article 31, 2014.

[24] J. A. Kim, H. S. Park, K. I. Park et al., "Proteome analysis of the anti-inflammatory response of flavonoids isolated from Korean Citrus aurantium L. in lipopolysaccharide-induced L6rat skeletal muscle cells," The American Journal of Chinese Medicine, vol. 41, no. 4, pp. 901-912, 2013.

[25] K. A. Mirza, S. L. Pereira, N. K. Edens, and M. J. Tisdale, "Attenuation of muscle wasting in murine $\mathrm{C}_{2} \mathrm{C}_{12}$ myotubes by epigallocatechin-3-gallate," Journal of Cachexia, Sarcopenia and Muscle, vol. 5, no. 4, pp. 339-345, 2014.

[26] S. B. Ballak, R. T. Jaspers, L. Deldicque et al., "Blunted hypertrophic response in old mouse muscle is associated with a lower satellite cell density and is not alleviated by resveratrol," Experimental Gerontology, vol. 62, pp. 23-31, 2015.

[27] S. Poussard, A. Pires-Alves, R. Diallo, J.-W. Dupuy, and E. Dargelos, "A natural antioxidant pine bark extract, oligopin ${ }^{5}$, regulates the stress chaperone HSPB1 in human skeletal muscle cells: a proteomics approach," Phytotherapy Research, vol. 27, no. 10, pp. 1529-1535, 2013.

[28] Y. Kishida, S. Kagawa, J. Arimitsua et al., "Go-sha-jinki-Gan (GJG), a traditional Japanese herbal medicine, protects against sarcopenia in senescence-accelerated mice," Phytomedicine, vol. 22, no. 1, pp. 16-22, 2015.

[29] A. Frati, D. Landi, C. Marinelli et al., "Nutraceutical properties of chestnut flours: beneficial effects on skeletal muscle atrophy," Food and Function, vol. 5, no. 11, pp. 2870-2882, 2014.

[30] Y. Tanabe, S. Maeda, N. Akazawa et al., "Attenuation of indirect markers of eccentric exercise-induced muscle damage by curcumin," European Journal of Applied Physiology, vol. 115, no. 9, pp. 1949-1957, 2015.

[31] I. Boz, M. Belviranli, and N. Okudan, "Curcumin modulates muscle damage but not oxidative stress and antioxidant defense following eccentric exercise in rats," International Journal for Vitamin and Nutrition Research, vol. 84, no. 3-4, pp. 163-172, 2014.
[32] S.-Y. Wang, W.-C. Huang, C.-C. Liu et al., "Pumpkin (Cucurbita moschata) fruit extract improves physical fatigue and exercise performance in mice," Molecules, vol. 17, no. 10, pp. 11864-11876, 2012.

[33] T.-S. Yeh, C.-C. Huang, H.-L. Chuang, and M.-C. Hsu, "Angelica sinensis improves exercise performance and protects against physical fatigue in trained mice," Molecules, vol. 19, no. 4, pp. 3926-3939, 2014.

[34] M. D. Matsumura, G. S. Zavorsky, and J. M. Smoliga, "The effects of pre-exercise ginger supplementation on muscle damage and delayed onset muscle soreness," Phytotherapy Research, vol. 29, no. 6, pp. 887-893, 2015.

[35] B. Sung, S. Y. Hwang, M. J. Kim et al., "Loquat leaf extract enhances myogenic differentiation, improves muscle function and attenuates muscle loss in aged rats," International Journal of Molecular Medicine, vol. 36, no. 3, pp. 792-800, 2015.

[36] A. A. Raut, N. N. Rege, F. M. Tadvi et al., "Exploratory study to evaluate tolerability, safety, and activity of Ashwagandha (Withania somnifera) in healthy volunteers," Journal of Ayurveda and Integrative Medicine, vol. 3, no. 3, pp. 111-114, 2012.

[37] S. JiPing, "Antifatigue effect of aqueous extract of salvia in endurance training rats' skeletal muscle," International Journal of Physical Sciences, vol. 6, no. 11, pp. 2697-2700, 2011.

[38] H.-Y. Jung, A.-N. Lee, T.-J. Song et al., "Korean mistletoe (Viscum album coloratum) extract improves endurance capacity in mice by stimulating mitochondrial activity," Journal of Medicinal Food, vol. 15, no. 7, pp. 621-628, 2012.

[39] I. Nallamuthu, A. Tamatam, and F. Khanum, "Effect of hydroalcoholic extract of Aegle marmelos fruit on radical scavenging activity and exercise-endurance capacity in mice," Pharmaceutical Biology, vol. 52, no. 5, pp. 551-559, 2014.

[40] S. S. Li, Z. C. Chen, and C. H. Zhang, "Effect of Tao-HongSi-Wu-Tang, a traditional Chinese herbal medicine formula, on physical fatigue in mice," African Journal of Traditional, Complementary and Alternative Medicines, vol. 10, no. 1, pp. 6065, 2013.

[41] S. Tan, F. Zhou, N. Li et al., "Anti-fatigue effect of ginsenoside $\mathrm{Rbl}$ on postoperative fatigue syndrome induced by major small intestinal resection in rat," Biological \& Pharmaceutical Bulletin, vol. 36, no. 10, pp. 1634-1639, 2013.

[42] L.-Z. Huang, B.-K. Huang, Q. Ye, and L.-P. Qin, "Bioactivityguided fractionation for anti-fatigue property of Acanthopanax senticosus," Journal of Ethnopharmacology, vol. 133, no. 1, pp. 213-219, 2011.

[43] M. Vitadello, E. Germinario, B. Ravara, L. D. Libera, D. DanieliBetto, and L. Gorza, "Curcumin counteracts loss of force and atrophy of hindlimb unloaded rat soleus by hampering neuronal nitric oxide synthase untethering from sarcolemma," The Journal of Physiology, vol. 592, no. 12, pp. 2637-2652, 2014.

[44] A. Hernández-Santana, V. Pérez-Lõpez, J. M. Zubeldia, and M. Jiménez-Del-Rio, "A rhodiola rosea root extract protects skeletal muscle cells against chemically induced oxidative stress by modulating heat shock protein 70 (HSP70) expression," Phytotherapy Research, vol. 28, no. 4, pp. 623-628, 2014.

[45] Y.-H. Lee, D.-H. Kim, Y. S. Kim, and T.-J. Kim, "Prevention of oxidative stress-induced apoptosis of $\mathrm{C} 2 \mathrm{C} 12$ myoblasts by a Cichorium intybus root extract," Bioscience, Biotechnology and Biochemistry, vol. 77, no. 2, pp. 375-377, 2013.

[46] E. Dargelos, C. Brulé, P. Stuelsatz et al., "Up-regulation of calcium-dependent proteolysis in human myoblasts under acute oxidative stress," Experimental Cell Research, vol. 316, no. 1, pp. 115-125, 2010. 
[47] Y. Nakashima, I. Ohsawa, K. Nishimaki et al., "Preventive effects of Chlorella on skeletal muscle atrophy in muscle-specific mitochondrial aldehyde dehydrogenase 2 activity-deficient mice," $B M C$ complementary and alternative medicine, vol. 14, article 390, 2014.

[48] S. E. Alway, B. T. Bennett, J. C. Wilson et al., "Green tea extract attenuates muscle loss and improves muscle function during disuse, but fails to improve muscle recovery following unloading in aged rats," Journal of Applied Physiology, vol. 118, no. 3, pp. 319-330, 2015.

[49] N. Kawanishi, K. Kato, M. Takahashi et al., "Curcumin attenuates oxidative stress following downhill running-induced muscle damage," Biochemical and Biophysical Research Communications, vol. 441, no. 3, pp. 573-578, 2013.

[50] J. Rodriguez, H. Gilson, C. Jamart et al., "Pomegranate and green tea extracts protect against ER stress induced by a high-fat diet in skeletal muscle of mice," European Journal of Nutrition, vol. 54, pp. 377-389, 2014.

[51] J. R. Jackson, M. J. Ryan, and S. E. Alway, "Long-term supplementation with resveratrol alleviates oxidative stress but does not attenuate sarcopenia in aged mice," Journals of Gerontology. Series A Biological Sciences and Medical Sciences, vol. 66, no. 7, pp. 751-764, 2011.

[52] B. T. Bennett, J. S. Mohamed, and S. E. Alway, "Effects of resveratrol on the recovery of muscle mass following disuse in the plantaris muscle of aged rats," PLoS ONE, vol. 8, no. 12, Article ID e83518, 2013.

[53] G. Gutierrez-Salmean, T. P. Ciaraldi, L. Nogueira et al., "Effects of (-)-epicatechin on molecular modulators of skeletal muscle growth and differentiation," The Journal of Nutritional Biochemistry, vol. 25, no. 1, pp. 91-94, 2014.

[54] C. D. Black, M. P. Herring, D. J. Hurley, and P. J. O'Connor, "Ginger (Zingiber officinale) reduces muscle pain caused by eccentric exercise," The Journal of Pain, vol. 11, no. 9, pp. 894903, 2010

[55] K. L. Pumpa, K. E. Fallon, A. Bensoussan, and S. Papalia, "The effects of Panax notoginseng on delayed onset muscle soreness and muscle damage in well-trained males: a double blind randomised controlled trial," Complementary Therapies in Medicine, vol. 21, no. 3, pp. 131-140, 2013.

[56] R. A. Shanely, D. C. Nieman, K. A. Zwetsloot et al., "Evaluation of Rhodiola rosea supplementation on skeletal muscle damage and inflammation in runners following a competitive marathon," Brain, Behavior, and Immunity, vol. 39, pp. 204-210, 2014.

[57] S. Choquette, T. Dion, M. Brochu, and I. J. Dionne, "Soy isoflavones and exercise to improve physical capacity in postmenopausal women," Climacteric, vol. 16, no. 1, pp. 70-77, 2013.

[58] M. Z. Allouh, "Effect of Ferula hermonis root extract on rat skeletal muscle adaptation to exercise," Experimental Biology and Medicine, vol. 236, no. 12, pp. 1373-1378, 2011.

[59] E. Vazeille, L. Slimani, A. Claustre et al., "Curcumin treatment prevents increased proteasome and apoptosome activities in rat skeletal muscle during reloading and improves subsequent recovery," The Journal of Nutritional Biochemistry, vol. 23, no. 3, pp. 245-251, 2012.

[60] M. Terauchi, N. Horiguchi, A. Kajiyama et al., "Effects of grape seed proanthocyanidin extract on menopausal symptoms, body composition, and cardiovascular parameters in middle-aged women: a randomized, double-blind, placebo-controlled pilot study," Menopause, vol. 21, no. 9, pp. 990-996, 2014.
[61] C. D. Black and P. J. O'Connor, "Acute effects of dietary ginger on muscle pain induced by eccentric exercise," Phytotherapy Research, vol. 24, no. 11, pp. 1620-1626, 2010.

[62] N. Alamdari, P. O’Neal, and P.-O. Hasselgren, "Curcumin and muscle wasting - a new role for an old drug?” Nutrition, vol. 25, no. 2, pp. 125-129, 2009.

[63] A. Shehzad and Y. S. Lee, "Molecular mechanisms of curcumin action: signal transduction," BioFactors, vol. 39, no. 1, pp. 27-36, 2013.

[64] M. Peleli, I.-K. Aggeli, A. N. Matralis, A. P. Kourounakis, I. Beis, and C. Gaitanaki, "Evaluation of two novel antioxidants with differential effects on curcumin-induced apoptosis in C2 skeletal myoblasts; involvement of JNKs," Bioorganic and Medicinal Chemistry, vol. 23, no. 3, pp. 390-400, 2015.

[65] H. Kim, T. Suzuki, K. Saito et al., "Effects of exercise and tea catechins on muscle mass, strength and walking ability in community-dwelling elderly Japanese sarcopenic women: a randomized controlled trial," Geriatrics \& Gerontology International, vol. 13, no. 2, pp. 458-465, 2013.

[66] G. Howatson and K. A. van Someren, "The prevention and treatment of exercise-induced muscle damage," Sports Medicine, vol. 38, no. 6, pp. 483-503, 2008.

[67] G. L. Warren, D. A. Lowe, and R. B. Armstrong, "Measurement tools used in the study of eccentric contraction-induced injury," Sports Medicine, vol. 27, no. 1, pp. 43-59, 1999.

[68] K. Cheung, P. A. Hume, and L. Maxwell, "Delayed onset muscle soreness: treatment strategies and performance factors," Sports Medicine, vol. 33, no. 2, pp. 145-164, 2003.

[69] L. Hirose, K. Nosaka, M. Newton et al., "Changes in inflammatory mediators following eccentric exercise of the elbow flexors," Exercise Immunology Review, vol. 10, pp. 75-90, 2004.

[70] P. M. Clarkson and M. J. Hubal, "Exercise-induced muscle damage in humans," American Journal of Physical Medicine \& Rehabilitation, vol. 81, no. 11, pp. S52-S69, 2002. 


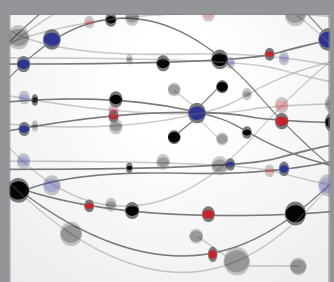

The Scientific World Journal
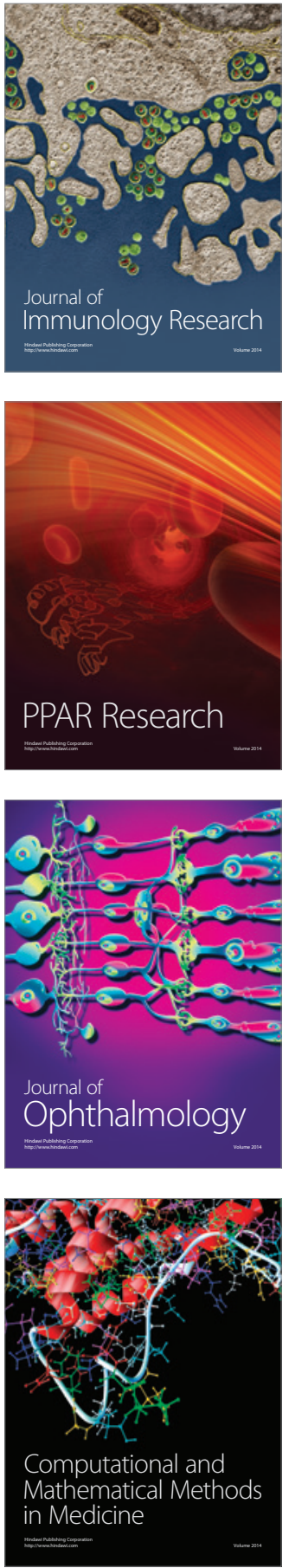

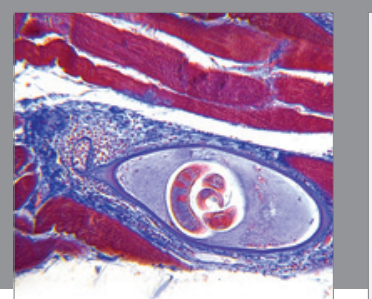

Gastroenterology Research and Practice

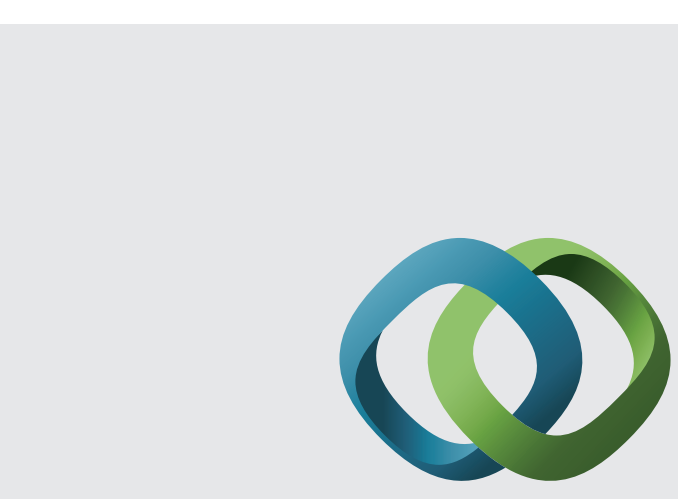

\section{Hindawi}

Submit your manuscripts at

http://www.hindawi.com
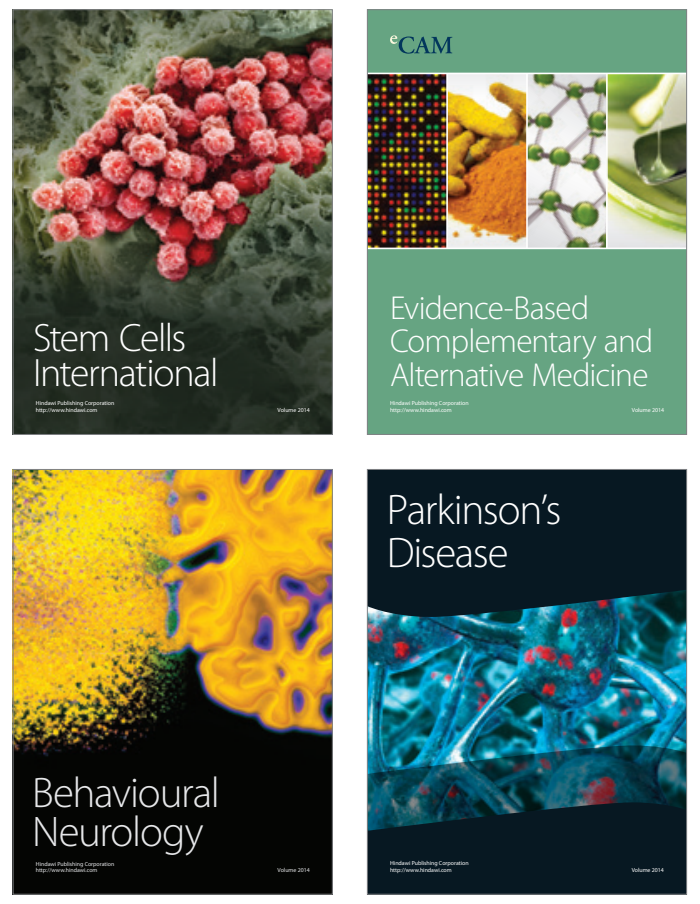
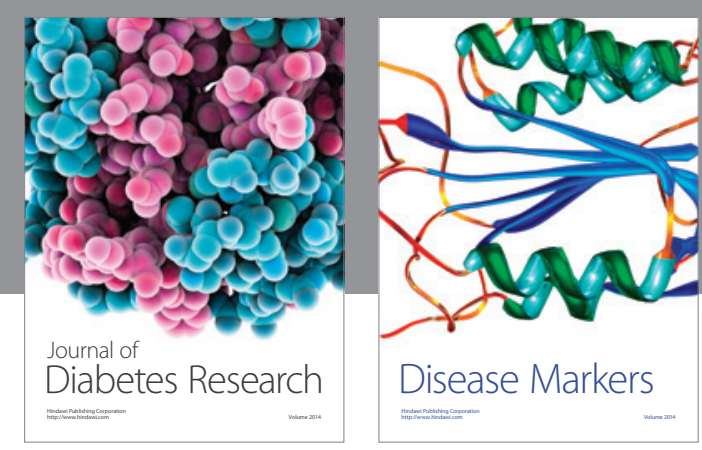

Disease Markers
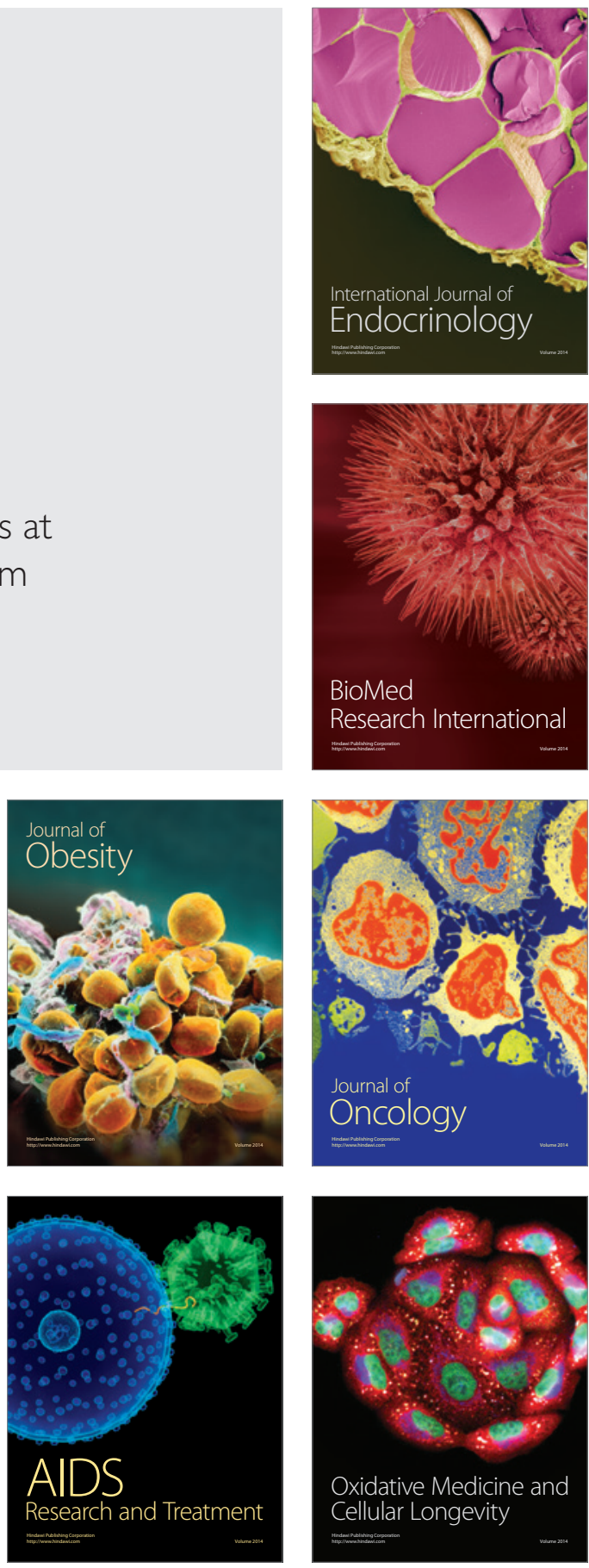